\title{
Anabases
}

ANABASES Traditions et réceptions de l'Antiquité

$27 \mid 2018$

Varia

\section{Jérôme Carcopino et la mémoire perdue de M. Valerius Messalla Corvinus (cos. 31 av. J.-C.)}

\section{Cyrielle Landrea}

\section{(2) OpenEdition}

1 Journals

Édition électronique

URL : http://journals.openedition.org/anabases/7147

DOI : 10.4000/anabases.7147

ISSN : 2256-9421

Éditeur

E.R.A.S.M.E.

Édition imprimée

Date de publication : 1 avril 2018

Pagination : 177-184

ISSN : 1774-4296

\section{Référence électronique}

Cyrielle Landrea, « Jérôme Carcopino et la mémoire perdue de M. Valerius Messalla Corvinus (cos. 31 av. J.-C.) », Anabases [En ligne], 27 | 2018, mis en ligne le 01 avril 2020, consulté le 20 janvier 2021. URL : http://journals.openedition.org/anabases/7147 ; DOI : https://doi.org/10.4000/anabases.7147

(c) Anabases 


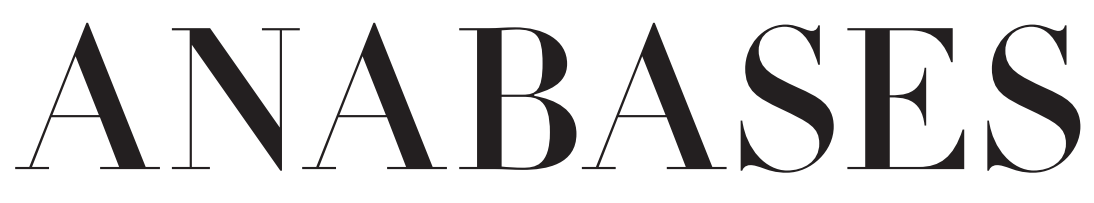

Traditions et Réceptions de l'Antiquité

$$
\begin{aligned}
& \text { No27 } \\
& 2018
\end{aligned}
$$

E.R.A.S.M.E.

Université Toulouse - Jean Jaurès 



\section{Sommaire}

$\mathrm{N}^{\circ} 27-2018$

\section{Historiographie et identités culturelles}

\section{Sébastien Cazalas}

“ et pour ce recite Valere... ». La place de l'Antiquité dans l'arsenal

intellectuel d'un grand prélat français du XVe siècle :

Jean Juvénal des Ursins (I388-I473) . . . . . . . . . . . . . . . . . . . II

Loic Marcou

La réception de l'Antiquité dans l'œuvre de la femme de lettres grecque

Rhéa Galanaki. . . . . . . . . . . . . . . . . . . . . .

\section{Traditions du patrimoine antique}

« Restituer Herculanum II. Des archives de fouilles aux restitutions 3D

Alexandra DARDENAY

Introduction : restituer l'espace domestique à Herculanum

grâce aux outils informatiques de reconstruction virtuelle ;

enjeux et problèmes. . . . . . . . . . . . . . . 4

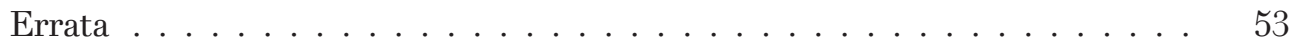

Carla Marotta

L’utilisation des données archivistiques dans l'enquête archéologique : nouvelles découvertes sur la maison d'Argos . . . . . . . . . . . . . . .

Emmanuelle Rosso

Restituer l'emplacement des statues dans les édifices d'Herculanum?

Problèmes de méthode . . . . . . . . . . . . . . . 7I 
Hélène Eristov, Marie-Laure Maraval

Restituer les décors perdus de la maison de Neptune et d'Amphitrite :

enquête, méthodes, résultats . . . . . . . . . . . . 9 9I

Maud Mulliez

Restauration numérique des peintures murales de la maison

de Neptune et Amphitrite à Herculanum : de l'expérimentation

matérielle à l'intégration des données dans un modèle 3D . . . . . . . Io3

\section{Archéologie des savoirs}

Nicolas SIRON

L'histoire de Philippidès d'Hérodote à Lucien.

Une incursion dans l'atelier du mythe . . . . . . . . . . . . . . . . Iog

Constantin Raios

Le coracin (xo@axîvos) du lac de Tibériade (F. Josèphe, B.J. III, 520) . . .

\section{Actualités et débats}

Claude Azıza

Antiquités parallèles (8). Le syndrome du sein droit . . . . . . . . . . . . I6I

Elisabeth DÉcultot

Winckelmann. Moderne Antike / Winckelmann. Modern Antiquity.

Exposition, Weimar, Neues Museum, 7 avril-2 juillet 20I7 . . . . . . . I67

\section{Lire, relire la bibliothèque des sciences de l'Antiquité}

Cyrielle LANDrea

Jérôme Carcopino et la mémoire perdue

de M. Valerius Messalla Corvinus (cos. 3г av. J.-C.) . . . . . . . . . . . . $\quad$ I77

Jérôme CARCopino

Notes biographiques sur M. Valerius Messala Corvinus . . . . . . . . . I I85

\section{L'atelier de l'histoire : chantiers historiographiques}

L'Atelier des doctorants (coordonné par Adeline Grand-Clément) (15)

Jonathan Pérez Mostazo

Cantabri aut vascones. La reception de la Antigüedad

en la cultura histórica vasca del siglo XIX . . . . . . . . . . . . . . . . . 209 


\section{Comptes rendus}

William H.F. Altman (éd.)

Brill's Companion to the Reception of Cicero (A. A. Raschieri). . . . . . . . 2 2I9

Anthony A. Barrett, Elaine Fantham, John C. Yardley

The Emperor Nero. A Guide to the Ancient Sources (É. Deniaux) . . . . . . 22I

Pascale Barthélémy et Violaine sébillotte cuchet (éd.)

Clio. Femmes, Genre, Histoire $\mathrm{n}^{\circ} 43$ : Citoyennetés (M. L. Napolitano) . . . 222

Frédéric Colin, Olivier Huck, Sylvie Vanseveren (éd.)

Interpretatio. Traduire l'altérité culturelle dans les civilisations

de l'Antiquité (Cl. Joncheray) . . . . . . . . . . . . . . . . . . . . 224

Angus Fletcher

Comic Democracies. From Ancient Athens

to the American Republic (A. de Crémoux). . . . . . . . . . . . . . . . . . 226

David Hernández de la Fuente

El despertar del alma. Dioniso y Ariadna: mito y misterio (A. Iriarte) . . . . 228

Ted Kaizer (éd.)

Religion, Society and Culture at Dura-Europos (P. G. Michelotto) . . . . . 229

Jacques Le Goff, Jean-Pierre Vernant

Dialogue sur l'histoire. Entretiens avec Emmanuel Laurentin (C. Lucci). . 23I

Justine Mac Connell, Edith Hall

Ancient Greek Myth in World Fiction since ig8g (A. de Crémoux) . . . . . . 233

Angelo Mazzocco, Marc Laureys (éd.)

ANew Sense of the Past. The Scholarship

of Biondo Flavio (I3g2-I463) (C. Lucci). . . . . . . . . . . . . . . . . . . . . . 234

Seth L. Schein

Homeric Epic and Its Reception. Interpretative Essays (S. Sistac) . . . . . . 236

Arbogast SснмiтT

Wie aufgeklärt ist die Vernunft der Aufklärung?

Eine Kritik aus aristotelischer Sicht (L. Calvié) . . . . . . . . . . . . . . . . 238

Richard Sorabu (éd.)

Aristotle Re-Interpreted. New Findings on Seven Hundred Years

of the Ancient Commentators (P. Butti de Lima) . . . . . . . . . . . . . . . 239

Laura SwiFT

Greek Tragedy. Themes and Contexts (G. Aujac) . . . . . . . . . . . . . . 24

Zara Martirosova TorLone

Vergil in Russia. National Identity and Classical Reception

(D. Millet-Gérard) . . . . . . . . . . . . . . . . . . 243 
Jürgen von UNGERN-STERNBERG

Les chers ennemis. Deutsche und französische

Altertumswissenschaftler in Rivalität und Zusammenarbeit (L. Calvié) . . 2444

Robert W. WALLACE

Reconstructing Damon: Music, Wisdom Teaching, and Politics

in Perikles'Athens (A. Brancacci) . . . . . . . . . . . . . . . . . . . . . 246

T. P. Wiseman

The Roman Audience : Classical Literature as Social History (C. Landrea) 249

Résumés . . . . . . . . . . . . . . . . . . . . . . . . . . 253

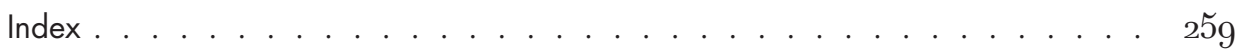




\section{Lire, relire la bibliothèque des sciences del'Antiquité}



Anabases 27 (2018), p. 177-184.

\section{Jérôme Carcopino et la mémoire perdue de M. Valerius Messalla Corvinus (cos. 31 av. J.-C.)}

Cyrielle LANDrea

a monumentalité de l'œuvre de J. Carcopino est indéniable et présenter succinctement sa carrière serait une gageure'. L'article “ Notes biographiques sur M. Valerius Messala Corvinus (64 av. J.-C. - 8 ap. J.-C.) » est paru en 1946 dans la Revue de philologie, de littérature et d'histoire ancienne et se place à un moment-clé de la vie de l'auteur, puisque J. Carcopino avait été non seulement révoqué de ses fonctions à la Libération, mais également traduit devant la Haute Cour pour sa participation au gouvernement de Vichy. Emprisonné à Fresnes, il avait ensuite obtenu sa libération provisoire en février 1945. Il dut toutefois attendre 1947 pour obtenir un non-lieu. Les années 19461947 furent logiquement les moins prolifiques ${ }^{2}$ et cette contribution est atypique dans l'œuvre du savant. Si J. Carcopino appréciait effectivement les biographies de grands personnages ${ }^{3}$, cet article est sa seule monographie d'un personnage réputé secondaire.

De prime abord M. Valerius Messalla Corvinus (cos. 31 av. J.-C.) est beaucoup moins connu que ses contemporains, tels Agrippa, Mécène et a fortiori Octavien-

1 Il fut notamment professeur à la Sorbonne, directeur de l'Ecole française de Rome (1937-1940), secrétaire d'État à l'Education nationale du maréchal Pétain (1941-1942) et membre de l'Académie française.

2 Son ouvre comporte 338 travaux divers et seulement deux sont parus en 1946, cf. G. Charles-Picard, J. Heurgon et W. Seston (dir.), Mélanges d'archéologie, d'épigraphie et d'histoire offerts à J. Carcopino, Paris, 1966, p. 1-23.

3 Il suffit ici par exemple de mentionner Sylla ou la monarchie manquée (Paris, 1931). 
Auguste. Pourtant il fait partie de la prestigieuse gens Valeria, une des gentes maiores. Selon une opinion communément admise, ces lignées patriciennes sont les plus anciennes et certaines prétendent même descendre des premiers sénateurs de Rome. Au-delà du prestige et du capital symbolique de la lignée des Valerii, le parcours de Mesalla Corvinus est singulier, car il s'inscrit dans une période d'intenses mutations entre la fin de la République et l'émergence du Principat. Messalla a successivement embrassé toutes les convictions politiques de son temps. À la mort de César, il avait à peine vingt ans. Disciple de Cicéron et ami de Brutus, ses convictions républicaines en firent un proscrit ${ }^{4}$. Après la bataille de Philippes, en 42 av. J.-C., Messalla rallia Antoine. En 36 av. J.-C., lieutenant d'Antoine, il fut "prêté » à Octavien pour vaincre Sextus Pompée selon les clauses des accords de Tarente. En 31 av. J.-C., il est consul et s'engage auprès d'Octavien dans la bataille navale d'Actium. Enfin son attitude est plus ambiguë à l'égard du Principat, abdiquant la préfecture urbaine en 26 av. J.-C. après seulement six jours d'exercice, mais acceptant le titre de curateur des eaux et honorant Auguste du titre de Pater patriae en 2 av. J.-C. Fin lettré, écrivain à ses heures, il est également connu pour avoir animé un cercle littéraire de la même ampleur que celui de Mécène.

Le propos de J. Carcopino s'appuie comme toujours sur une grande érudition et une parfaite connaissance des sources antiques, surtout littéraires. En outre, il put compter sur la notice de la Prosopographia imperii romani $\left(P R^{1}\right.$ $\mathrm{V} 90)^{5}$, sur de grandes monographies du XIX ${ }^{\mathrm{e}} \mathrm{s}^{6}{ }^{6}$ et sur l'ouvrage de J. Hammer, alors dernière étude d'envergure ${ }^{7}$. J. Carcopino fut attiré par la “notoriété littéraire » du personnage qu'il avait dû rencontrer à de maintes reprises dans ses études virgiliennes ${ }^{8}$ et plus largement dans ses travaux sur les poètes

4 Appien, Guerres civiles V, 12, 113.

5 La notice de la Realencyclopädie n'était pas encore parue (R. HansLik, 1955, s. v. "Valerius" n 261, RE, VIII A1, 1955, col. 131-157.

6 Les plus importantes sont celles de L. WieseDeM. Val. Messallae Corviniuitaet studiis doctrinae, Berlin, 1829, M. J. Valeton M. Valerius Messala Corvinus, Groningen, 1874, L. Fontaine De Valerio Messala Corvino, Paris, 1878 et H. Schulz De M. Valerii Messallae aetate, Stettin, 1886.

7 J. Hammer (Prolegomena to an edition of the Panegyricus Messalae. The military and political Career of M. Valerius Messala Corvinus, New York, 1925). L'ouvrage est très largement cité par J. Carcopino.

8 Dès 1919 paraissait Virgile et les origines d'Ostie. Le Catalepton souvent attribué à Virgile fit aussi l'objet de plusieurs études, notamment : “Vergiliana - I. À propos du Catalepton», RPh 46 (1922), p. 156-184 et “Encore le Catalepton: réponse à M. E. Galletier », RPh 53 (1927), p. 84-89. Messalla y est souvent présenté comme un inspirateur des vers virgiliens. 
d'époque augustéenne qui dédièrent de nombreux vers au patricien. La mémoire perdue de Messalla est d'emblée signalée, en pointant le paradoxe entre sa renommée antique et la perte des écrits du patricien (p. 96). Sa formation et ses relations avec Cicéron le prédisposaient aussi à la rhétorique et à des domaines éclectiques : éloquence, poésie élégiaque et érotique, littérature antiquaire, histoire, grammaire, sans oublier la rédaction de Mémoires. En outre Les Secrets de la correspondance de Cicéron parurent en 1947 ; nul doute que les travaux préparatoires lui permirent encore de rencontrer ce disciple de Cicéron qualifié de fulgentissimus iuuenis ${ }^{9}$.

Les Modernes se sont pourtant globalement désintéressés de cette figure patricienne, à l'exception de l'homme de lettres. À contre-courant J. Carcopino étudie ici l'homme et son parcours, en se penchant sur les problèmes d'établissement de la carrière du patricien dont il livre une chronologie commode en fin d'article (p. 117) ${ }^{10}$. Il passe rapidement sur les événements faisant l'unanimité dans la communauté savante (p. 97-98), comme l'exercice du consulat en 31 av. J.-C. ou la célébration d'un triomphe en 27 av. J.-C. Effectivement, plusieurs problèmes accaparent ici l'attention, comme le changement d'allégeance (p. 99-108), l'expédition contre les Salasses, la guerre illyrienne (p. 108-113) et l'utilisation des écrits de Messalla par les autres auteurs, comme le livre V des Guerres civiles d'Appien inspiré des Histoires de Messalla" ${ }^{11}$

La datation de l'allégeance à Octavien est loin de faire consensus. J. Carcopino remet d'abord en cause la datation haute proposée parJ. Hammer, selon ce dernier Messalla se serait détaché de l'entourage d'Antoine dès la fin des années $40^{12}$

9 Velleius Paterculus, Histoire romaine II, 71, 1.

10 J. Carcopino a consacré de nombreux articles à des points chronologiques controversés, notamment : " La date de la mort de Caius Gracchus », BSNAF (1930), p. 112-113 et « Sur la date de la naissance de César », CRAI 77-3 (1933), p. 377-378.

1 Cet emploi magnifia le rôle pourtant mineur du patricien durant la guerre de Sicile : “Ces détails propres à grandir notre homme, l'importance donnée au sauvetage d'Octave en péril, et l'insistance avec laquelle Appien a fait un sort aux moindres allées et venues de Messala » (p. 100).

12 J. Hammer, Prolegomena, p. 24-27. Cette thèse est notamment reprise par H. Zehnacker (1983, p. 62) dans son édition CUF du tome 33 de Pline l'Ancien. Selon l'auteur, le séjour alexandrin, après l'entrevue de Tarse, aurait profondément choqué Messalla. Le mois de septembre 40 aurait marqué son retour à Rome et son ralliement à la cause octavienne. Plus récemment P. TANSEy « Messalla Corvinus and the Bellum Siculum ", Latomus 66 (2007), p. 888) accrédite aussi l'hypothèse d'une adhésion antonienne très courte (41-40 av. J.-C.). 
ou peu avant $38^{13}$. Le patricien se serait donc rapidement rendu compte que le projet politique d'Antoine n'aurait pas été la restauration de la République. D'autres hypothèses placent le ralliement juste avant la guerre de Sicile en $36^{\mathbf{1 4}}$, ce que réfute l'auteur : “ Octave, tout en traînant Messala après lui, avait voulu l'éloigner de toute fonction militante et véritablement active » (p. 103). Il ajoute même qu'Octavien avait peu confiance en lui. Ensuite c'est grâce à une approche comparatiste que J. Carcopino opta pour le ralliement à Octavien en 33. Il met alors en parallèle le parcours de Messalla et celui de L. Sempronius Atratinus (cos. 34 av. J.-C.). Lors de la paix de Brindes, Atratinus devint augure, à l'âge de trente-trois ans et cette promotion constituait une première étape en vue du consulat, qui fut envisagé dès la paix de Pouzzoles de 39 et devint effectif cinq années plus tard. Messalla géra quant à lui le consulat à l'âge de trente-trois ans. En étudiant la carrière d'Atratinus, J. Carcopino a cru pouvoir dégager une règle “ de l'année d'intervalle » qui s'appliquerait aussi à la carrière de Messalla. La désignation en qualité d'augure précéderait alors d'une année celle au consulat. Par conséquent le patricien serait devenu augure en 33 av. J.-C. Cette hypothèse fondée sur un parallélisme de carrière paraît bien fragile ${ }^{15}$. Quoi qu'il en soit, le changement d'allégeance est lourd de sens ${ }^{16}$. Au-delà des visions d'un “Talleyrand antique », l'idée du service de la res publica transparaît ${ }^{17}$. Le choix du meilleur parti et du camp le plus juste induit une réflexion sur la conception même de la res publica. Octavien représentait alors au mieux le respect de cette dernière.

13 Comme semblerait l'attester un passage d'Horace (Sat. I, 10, 28) faisant allusion à l'activité judiciaire de Messalla. Deux objections peuvent être formulées. D'abord ceci est entièrement dissociable du changement d'allégeance, puisque la date de 36 , voire peu après, est préférable pour la rédaction des Satires. En outre, un ralliement peu avant 38 est peu crédible, car aucun enjeu contemporain ne peut expliquer ce revirement.

14 Pour A. E. Glauning Die Anhängerschaft des Antonius und des Octavian, Leipzig, 1936, p. 16, la débauche inacceptable d'Antoine aurait été l'élément déclencheur de l'allégeance à Octavien. La date de 36 est encore maintenant largement présente dans une historiographie dominée par R. Syme The Augustan Aristocracy, Oxford, 1986, p. 202.

15 Cette thèse est reprise ultérieurement par l'auteur, arguant que l'octroi de l'augurat ne peut pas avoir lieu avant les derniers mois de 34, mais il ne remet pas en avant l'argument du parallélisme entre les deux carrières (CARcopino, Les secrets, p. 345).

16 Concernant les attitudes à l'égard d'Auguste et les changements d'allégeance, voir notamment: J. L. Butrica, "Messalla and the Principate », in C. Deroux (dir.), Studies in Latin Literature and Roman History, VII, Bruxelles, 1994, p. 279-296.

17 Plutarque, Vie de Brutus LIII, 3 : « J'ai toujours été, César, du parti le meilleur et le plus juste ». 
Après la question de l'allégeance, c'est celle de la lutte contre les Salasses qui est au cœur des analyses : “Celle-ci, il est vrai, n’a pas été comprise par les modernes biographes de Messala, lesquels n'ont su la localiser, ni sur la carte, ni sur le calendrier»(p.108). Contrairement à une opinion communément admise, l'historien ne les identifie pas aux Salasses du val d'Aoste et opte pour une bipartition de ce peuple: les Salasses gaulois et ceux d'Illyrie. Les Salasses d'Appien seraient ainsi différents de ceux mentionnés par Strabon ${ }^{18}$. J. Carcopino forge donc une nouvelle hypothèse, selon laquelle Messalla aurait eu affaire aux deux tribus (p. 109). L'historiographie est majoritairement hostile à ce raisonnement ${ }^{19}$; selon A. Momigliano, J. Carcopino a inutilement compliqué le problème ${ }^{20}$. Cette hypothèse est néanmoins soutenue par des disciples de J. Carcopino, comme P. Grimal ${ }^{21}$.

La datation de l'expédition est un autre sujet de discorde. À la fin des années 30 av. J.-C., les Salasses multiplièrent les pillages et les actes d'insoumission. Cependant l'attention d'Octavien est alors accaparée par les préparatifs guerriers contre Cléopâtre et Antoine. L’envoi de Messalla aurait été dans cette perspective postérieur à la bataille d'Alexandrie en 30 (p. 112) et au proconsulat de Syrie. Ainsi l'expédition contre les Salasses peut-elle être située à partir de 29,

18 Les Salasses de Strabon disposent de gisements aurifères (IV, 6, 7), alors que les Salasses d'Appien ont des gisements de sel (Le livre illyrien XVII). À partir de ce constat, J. Carcopino croit en la bipartition de ce peuple.

19 Cf. W. Schmitthenner, “ Octavians militärische Unternehmungen in den Jahren 35-33 v. Chr. », Historia 7 (1958), p. 234-236. R. Syme juge même cette distinction aberrante (The Augustan, p. 204).

20 A. Momigliano, “Panegyricus Messallae and Panegyricus Vespasiani », JRS 40 (1950), p. 40. Cette complication est due à sa théorie sur la non-intervention de Messalla dans les opérations en Pannonie en 35-34. La théorie repose sur le fait qu'il était alors en Orient avec Antoine. D'ailleurs l'examen d'un passage de Pline l'Ancien attesterait la caducité des allégations de J. Carcopino. Pline mentionne l'inscription du trophée de la Turbie (III, 136-137) où tous les peuples alpins soumis par Auguste sont énumérés. Parmi eux figurent les Salasses. Cela n'aurait pas de sens si les Salasses avaient été un peuple illyrien.

21 P. Grimal “ Le roman de Délie et le premier livre des Elégies de Tibulle », REA 60 (1958), p. 134 et “ Les conséquences d'un cursus : Tibulle, Properce et Messalla », dans Rome : la littérature et l'histoire, Rome, 1986, p. 773) s'appuie clairement sur les travaux de J. Carcopino. J. Prieur « L’histoire des régions alpestres. Alpes Maritimes, Cottiennes, Graies et Pennines sous le Haut-empire romain $\mathrm{I}^{\mathrm{er}}-\mathrm{III} \mathrm{I}^{\mathrm{s}}$ s. ap. J.-C. », in W. HaAse (dir.), $A N R W$, II, 5, 2, Berlin et New York, 1976, p. 637 maintient également cette bipartition. 
peut-être lors de son proconsulat en Gaule de 28-2722 (donc pas en $34^{23}$ av. J.-C., en appendice à la guerre d'Illyrie). Les Salasses furent ensuite définitivement matés par Terentius Varro en 26 ou $25^{24}$.

La soumission des Salasses doit en fait être replacée dans le contexte plus large de pacification et d'organisation de l'Occident romain. D'ailleurs Messalla a-t-il participé à la campagne d'Octavien en Illyrie en 35-33 av. J.-C. ? Le Panégyrique de Messalla est la seule source mentionnant la participation à cette campagne ${ }^{25}$. J. Carcopino (p. 112-113) revient sur ce poème et note le silence d'Appien lorsqu'il cite les généraux accompagnant Octavien en Illyrie ${ }^{26}$. Ensuite un paradoxe émerge. Si Messalla était sous les ordres du triumvir, le panégyriste n'aurait pas pu autant le célébrer. En outre, si cette campagne était si brillante, elle contraste alors fortement avec celle menée ultérieurement contre les Salasses. J. Carcopino pense donc que Messalla ne participa pas au conflit illyrien et qu'après la guerre de Sicile, il rejoignit Antoine à Alexandrie (p. 113).

Par ses prises de position indéniables et quelquefois contestables, cet article a suscité à la fois admiration ${ }^{27}$ et contestation, nourrissant jusqu'à maintenant de multiples études et critiques. R. Syme a résumé cette attitude ambivalente : "The article, questionable in other assertions, was styled 'brilliant' by A. Momigliano „28. Même si l'auteur britannique constate la grande érudition de

22 Cette hypothèse est aussi suivie par Syme, The Augustan, p. 204.

23 Cette erreur chronologique fut très largement suivie, notamment par J. Prieur, "L'histoire », 1976, p. 637 et G. Walser, Summus Poeninus. Beiträge zur Geschichte des grossen St. Bernhard-Passes in römischer Zeit, Wiesbaden, 1984. p. 15 (en 34-33 av. J.-C.).

24 Dion Cassius, Histoire romaine LIII, 25, 1.

25 Panégyrique de Messalla, 107-110. J. Наммer Prolegomena, p. 35-40 croit en la participation de Messalla, comme de nombreux historiens. C'est encore le cas de N. Biffi, “Messalla fra Antonio e Ottaviano », Orpheus 15-2 (1994), p. 468-469. La participation de Messalla à la campagne illyrienne aurait été un acte marquant, préludant au consulat et au ralliement à Octavien.

26 Appien, Les guerres d'Illyrie XX.

27 C'est notamment le cas de son disciple P. Grimal Tibulle : livre I, Paris, 1967, p. 4 qui ne cache pas son admiration notamment pour les travaux sur Messalla, la fixation de la chronologie des élégies déliennes et l'étude des relations entre Messalla et Tibulle. P. Grimal insiste souvent sur l'importance de la contribution de J. Carcopino, par exemple dans “ Les conséquences d'un cursus », p. 773 : “Constatons d'abord que, malgré quelques objections de détail, qui, d'ailleurs, ne concernent guère notre problème, les grandes lignes du cursus de M. Valerius Messalla Corvinus demeurent, depuis le travail de M. Carcopino, solidement établies ».

28 Syme, The Augustan, p. 204 (note 29). 
J. Carcopino, celle-ci aurait été au service de thèses contestables. En outre, c'est justement cette érudition qui aurait pu tromper les plus naïfs selon R. Syme ${ }^{29}$.

Cependant l'impact de cet article de Messalla Corvinus fut en fait limité. Ainsi dans les années 1950, R. Syme publiait-il le compte-rendu de l'ouvrage d'A. E. Gordon consacré à Potitus Messalla ${ }^{30}$, probable cousin de Messalla Corvinus. En analysant le regain de fortune des Valerï Messallae après une éclipse d'un siècle dans l'exercice du consulat (161-61 av. J.-C.), l'érudit anglais mentionne le parcours de Corvinus sans faire allusion à l'article de J. Carcopino, alors qu'il a fait part d'hypothèses issues de l'Histoire romaine et du Sylla ${ }^{31}$. Plus tard R. Syme cite brièvement l'article de J. Carcopino dans History in Ovid à propos de la question controversée du changement d'allégeance de Messalla Corvinus ${ }^{32}$. Il met alors en avant la vulnérabilité des hypothèses de l'érudit français.

L'œuvre monumentale intitulée Aufstieg und Niedergang der römischen Welt $(A N R W)$ a également consacré une étude à M. Valerius Messalla Corvinus (cos. 31 av. J.-C. $)^{33}$. A. Valvo commence par rappeler le renouveau historiographique considérable entre 1925 et 1955 . Toutefois il cite seulement l'ouvrage de J. Hammer et la notice de R. Hanslik dans la RE. L'article de J. Carcopino est alors rejeté plus loin dans une note de bas de page concernant les Mémoires du patricien ${ }^{34}$. La contribution de l'érudit français semble ainsi marginalisée dans un premier temps avant d'être citée à la fin de la présentation de l'historiographie contemporaine. L'établissement d'une chronologie systématique pour la vie de Messalla est particulièrement apprécié35 et l'article est ensuite abondamment cité.

29 Syme, The Augustan, p. 207: "Such is the thesis expounded in an erudite disquisition, which may have deceived the ingenuous ». L'auteur remet ici en cause la datation du changement d'allégeance de Messalla Corvinus et l'abandon d'Antoine.

30 A. E. Gordon, Potitus Valerius Messalla, consul suffect 29 B. C., Berkeley-Los Angeles, 1954.

31 R. Syme, “Potitus Valerius Messalla Consul Suffect 29 B. C. », JRS 45 (1955), p. $157-158$.

32 R. Syme, History in Ovid, Oxford, 1978, p. 120.

33 A. Valvo, “ M. Valerio Messalla Corvino negli studi più recenti », in W. HaAse (dir.), ANRW, II, 30, 3, Berlin et New York, 1983, p. 1663-1681.

34 A. Valvo, “ M. Valerio Messalla Corvino », p. 1664 (note 9).

35 A. VAlvo, “M. Valerio Messalla Corvino », p. 1669 : “Si deve a J. Carcopino una precisa, e per certi aspetti rivoluzionaria, sistemazione della cronologia relative alla carriera di Messalla in quel periodo; come altre volte è accaduto, le originali soluzioni proposte dall'insigne studioso francese sono divenute il termine di riferimento di tutti i contribute successive sui medesimi problemi ». 
Etudier la réception de l'article de J. Carcopino consiste aussi à traiter les silences. Les études, y compris récentes, consacrées au patricien omettent souvent le texte de l'érudit français. C'est le cas de nombreuses notices biographiques ${ }^{36}$. Toutefois le manque le plus étonnant est sans nul doute la notice consacrée à M. Valerius Messalla Corvinus parue dans la nouvelle version de la Prosopographia imperii romani $\left(P I R^{2} \mathrm{~V} 143\right)$ qui ne cite pas la contribution de J. Carcopino, y compris pour la nature de la participation aux guerres de Sicile et d'Illyrie ${ }^{37}$.

Quoi qu'il en soit, cet article demeure une référence pour toutes les personnes intéressées par la prosopographie, par cette période charnière entre la fin de la République et l'émergence du Principat et par l'adaptation de l'ancienne nobilitas à la nouvelle donne politique. En effet l'itinéraire de Messalla Corvinus est singulier. Ancien proscrit, partisan d'Antoine avant de rallier le camp octavien, il symbolise les hésitations des nobles qui cherchent avant tout à se maintenir dans les sphères du pouvoir.

\section{Cyrielle Landrea}

Prag d'Histoire ancienne à l'Université Lille-SHS

Domaine Universitaire du Pont de Bois 59650 Villeneuve-d'Ascq

cyrielle.landrea@orange.fr

36 Par exemple la notice consacrée à Messalla Corvinus par A. LA Penna, La letteratura latina del primo periodo augusteo (42-15 a. C.), Rome, 2013.

37 Par exemple M. Heil et K. Wachtel renvoient à Syme The Augustan p. 202-204 pour le Panégyrique de Messalla qui avait pourtant été largement commenté par J. Carcopino. Or la note 29 p. 204 cite l'article de J. Carcopino. La réception est donc ici indirecte. 


\title{
NOTES BIOGRAPHIQUES
}

\author{
SLR \\ H. VALERILS MESSALA CORVINLS
}

(64 AV. J.-C. -8 AP. J.-C.)

La notoriété littéraire de M. Valerius Messala Corvinus, considérable dans l'antiquité, n'est plus qu'un souvenir. Nous n'en saisissons qu'un reflet fugitif dans les vers que lui ont dédiés les plus illustres de ses contemporains : Tibulle ${ }^{(1)}$, Horace ${ }^{(2)}$, Ovide ${ }^{(3)}$. Ses a histoires ${ }^{(4)}$ sont perdues, comme sa traduction d'Hypéride ${ }^{(5)}$, ses rapports ${ }^{(6)}$, ses pamphlets ${ }^{(7)}$ et ses dissertations grammaticales ${ }^{(r)}$. De ses poèmes, élégiaques et érotiques, en grec et en latin, c'est à peine si nous distinguons les langues et les genres au travers des allusions du Catalepton ${ }^{(9)}$ et des lettres de Pline le Jeune (10). Quant à l'orateur dont Cicéron avait pressenti la maftrise ${ }^{(11)}$, avant que Tibère, adolescent, ne songeât à perfectionner sa propre éloquence en écoutant la sienne ${ }^{(12)}$, et que Quintilien ${ }^{(13)}$ puis Tacite ${ }^{(14)}$ vanteront encore à l'égal des plus grands, nous ne possédons plus que le titre d'un de ses plaidoyers de jeunesse : celui qu'il prononça contra Aufidiam, dans un procès de succession ${ }^{(15)}$, entre juin 44

(1) Cf. infra, p. 98, n. 3 .

(a) Cf. infra, p. 113-114

(3) Ov., Pont., I, 7, 29-3o. Je ne mentionne pas Virgile qui, à mon avis, n'est point l'auteur du Catalepton (cf. infra, n. 9).

(4) Cf., notamment, Suét., Aug., LXXIV, 2 : Valerius Messala tradit...

(5) Quint., X, 5, 2.

(6) Le De vectigalium Asiae constitutione cité par Charisius, p. 146, 34.

(7) Cf. infra, p. 116 .

(d) Quint., I, 7, 23 et IX, 4, 38 .

(9) Catal., is, i4 et suiv.

(10) Pline le Jeune, Ep., V, 3, 5.

(11) Cic., $A d B r .$, I, 15, 1 (entre le 17 et le 27 juillet 43 av. J.-C.).

(12) Suét., Tib., LXX, 1 : In oratione latina, secutus est Corvinum Messalam, quem dicendo senem adolescens observarat.

(13) Quint., X, 1, 113: Messala nitidus et candidus, et quodammodo praeferens in nobilitatem suam...

(14) Tac., De or., XVIII, a : Cicerone mitior Corvinus et dulcior et in rerbis nagis elaboratus.

(15) Quint., X, 1, 22 : Utilissimum... utrimque habitas legere actiones ut... Servit Sulpicii atque Messalae, quorum alter pro Aufilia, conlra dixit alter. 
NOTES BIOGRAPHIQUES SUR M. VALERIUS MESSALA CORVIYUS.

et janvier 43 avant Jésus-Christ (1). Autant dire que rien n'est parvenu jusqu'à nous des talents qui, avec la noblesse de son lignage et les honneurs dont il fut comblé, concoururent à faire sa grande renommée.

De ces honneurs, du reste, et des missions qu'ils ont sanctionnés, la liste est plus facile à dresser que le caractère à définir et l'ordre à reconstituer, et malgré les études diligentes dont elle a été l'objet à plusieurs reprises (2), la biographie du personnage s'enveloppe toujours de nombreuses obscurités.

Sans doute nous sommes exactement renseignés sur les dernières étapes que Messala a franchies : son consulat en 31 avant Jésus-Christ (3); son triomphe remporté e sur la Gaule» en 27 avant Jésus-Christ ( ${ }^{(4)}$; son élévation, en 26 avant Jésus-Christ, à la Préfecture de la Ville, que, par scrupule de conscience, il a abdiquée six jours après l'avoir revêtue (s); et, en 11 avant Jésus-Christ, sa nomination, en remplacement d'Agrippa, à l'administration des aqueducs, qu'il devait conserver jusqu'à sa mort, survenue en 8 après Jésus-Christ (6), et dans laquelle Auguste ne lui donnera de successeur que quatre ans plus tard (7). Enfin, preuve à la fois de son constant loyalisme envers l'empereur et de la faveur dont il n'a pas cessé de jouir à la Cour, c'est lui qui, en a avant Jésus-Christ, sur l'invitation déférente de ses pairs du Sénat, a proposé en leur nom de décerner à Auguste le titre de père de la Patrie ${ }^{(8)}$.

Mais si la critique a réussi à déterminer selon toute vraisemblance l'année de sa naissance (64 avant J.-C. $\left.{ }^{(9)}\right)$; si, grấce à la correspondance de Cicéron, nous sommes informés de son studieux séjour à Athènes, de

(1) Entre le retour de Messala, d'Athènes (cf. Cic., Ad Att., XV, 17, 2, 14 juin 4' av. J.-C.) et le départ, enregistré, le 4 janvier 43 av. J.-C., par Cicéron en sa $V I$ Philippique $(6,16)$ du contradicteur de Messala, Servius Sulpicius Rufus pour une legatio auprès d'Antoine, d'où il ne devait pas revenir (Phil., IX, 1, 1 et suiv.; cf. ibid., VIII, 7,22 ), en février 43 av. J.-C..

(s) Notamment Wiese, De M. Val. Messallae Corvini vita et studiis doctrinae, Diss. Berlin, 1829 ; Valeton, M. Valerius Messala Corvinus, Diss. Groningen, 187 ' ; Fontaine, De Valerio Messala Corvino, Paris, 1878 ; et Jacob Hammer, cf. infra p. 98, n. 4 .

(3) Vell. Pat. , II, 84, 1; Appien, B. C., IV, 38; Tac., Ann., XIII, 34, 1; Cass. Dio, L, 10, 1. Cf. C. I. L., I' ${ }^{2}$, p. 160.

(o) C. I. L., Is, p. 50 : . .a(nno) dccxxvi Corvinus procos. ex Gallia vii $K$ (alendas) Oct(obres); ef. ibid., p. 77 : Messala vii K(alendas) Oct(obres) triumphavit. Le triomphe est donc du 25 septembre 27 av. J.-C. Cf. Pais, Fast. Tr., I, p. 315.

(s) Tac., Ann., VI, 11, 4; Saint Jérỏme, Chron., a. Abr. 1991.

(o) Ainsi qu'il résulte d'Ov., Pont., I, 7. 27.

(7) Frontin, De aquis, 99 et 103.

(s) Cf. Suét., Aug., LVill, 2 : Is (Messala) mandantibus cunclus, etc., et 3 (Suétone résume ici le récit méme des Annales de Messala).

(9) Par élimination du texte de Tacite, $D_{e}$ or., XVII, 6, et en supposant chez Saint Jéróme, Chron., a. Abr. 1958 , une intervention a vec a. Abr. $19^{53}$, par confusion du consulat de 59 : Caesare et Bibulo $c 0(n) s(u l i b u s)$ avec celui de 64 av. J.-C. : Caesare et Figulo $c o(n) s(u$ libus). C'est maintenant l'opinion courante (cf. Schanz, Röm. Lit., 11,1 , p. 28-29), telle que l'avait dégagée Fontaine, op. cit., p. 103-111 et que laccepte Hammer (cf. infra, p. 98, n. 4 ), p. 80. 
son retour à Rome en juin 44 avant J.-G. (1) et de son ralliement à l'armée de Brutus en juillet 43 avant J.-C. (2); si, enfin et surtout, les auteurs anciens, confirmés par l'épigraphie, nous permettent de jalonner la fin de sa vie de repères assurés, ceux-ci ne sauraient suffire à nous orienter dans les complications de la période comprise entre son consulat de 31 avant J.-C. et son triomphe de 27 avant J.-C. ; et, auparavant, l'activité qu'il a déployée de 42 à 31 avant J.-G., c'est-à-dire entres les défaites à Philippes de Cassius et de Brutus, dont il avait épousé la cause, et son accession à la magistrature suprême de l'État, flotte toujours, en ses phases mouvementées, dans la plus troublante indécision(3); et si approfondies qu'aient été les enquêtes du plus récent de ses biographes, Jacob Hammer (4), les conclusions auxquelles elles ont abouti appellent moins quelques retouches de détail qu'une révision d'ensemble. Les quelques pages qui vont suivre n'ont d'autre ambition que d'en rassembler les éléments.

Suivant le résumé que nous en a transmis Appien(5), la carrière militaire et politique de Messala, depuis sa déroute a Philippes, dans l'automne de 42 avant J.-C., jusqu'à son triomphe du 25 septembre 27 avant J.-C., se déroule ainsi qu'il suit. Conscient de l'irrémédiable destruction des dernières forces républicaines, Messala obtient sa grâce des triumvirs et passe à leur service : au service d'Antoine, pour commencer; puis à celui d'Octave ${ }^{(6)}$, qui lui confère le consulat l'année où, avec son concours, il vaincra à Actium( (7); et finalement à celui d'Auguste, qui lui accorde les honneurs du triomphe neuf mois à peine après la consécration, sous le nom quasi divin qu'il transmettra à tous ses successeurs, de son régime impérial ${ }^{(8)}$.

Mais tout de suite apparaissent les lacunes de ce schéma et, de prime abord, nous nous heurtons, pour l'utiliser, à une double difficulté : celle de savoir le moment où Messala a abandonné le parti d'Antoine pour celui d'Octave; et celle de situer, sur la carte et dans la durée, la suite des campagnes guerrières qu'a couronnées son triomphe.

(1) Voir la lettre, en date du 14 juin 44 av. J.-C., de Cic., Ad Att., XV, $17,3$.

(3) Voir la lettre écrite entre le 17 et le 27 juillet 44 av. J.-C. par Cic., $\mathrm{Ad} B \mathrm{Br}$, I, 15,1 .

(3) Il me semble inutile de rechercher à quelle date et en quelle qualité Messala aurait réparé la Voie Latine. Les allusions de Tib., 1, 7, 57-63 et Martial, VIII, $3,3-6$ et $X, 2,9^{-1} 2$ ne recouvrent, à mon sens, qu'une initiative privée prise par Messala, comme par C. Calvisius Sabinus (C.I. L., X, 6901), en bordure de leurs domaines respectifs.

(6) Jacob Hammer, Prolegomena to an Edition of the Panegyricus Messalae, the nilitary and political career of M. Valerius Messala Corvinus, Diss. Columbia, New-York, 1925.

(s) Appien, B. C., IV, 38, 159 et suiv.

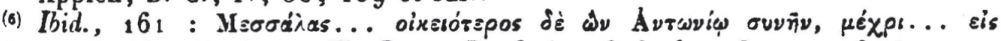

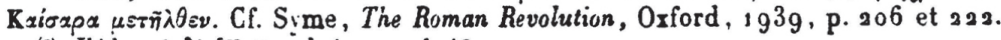

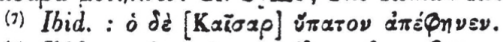

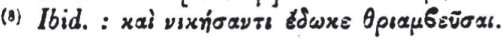


NOTES BLOGRAPHYQES SUR M. VALERIUS MESSALA CORVINUS.

Sur le premier point, les Modernes ont une tendance à faire remonter trop haut son changement d'allégeance. Par exemple, Fontaine (1) et Hammer (2), dont l'o pinion est généralement accréditée aujourd'hui, sont d'accord pour le dater de la guerre de Sicile, où, dans l'été de 36 avant J.-C., Octave, par un admirable coup double de sa fortune, s'est débarrassé, à quelques jours de distance, et de Sextus Pompée, par la défaite qu'il lui avait infligée à Nauloque, et de son collègue Lépide, dont it avait, peu après, débauché les troupes à Messine ${ }^{(3)}$. A première vue, les textes semblent leur donner raison, puisque, selon Appien et Dion Cassius, Octave n'eut qu'à se louer, durant cette double campagne navale et militaire, du concours de Messala. Mais, à la réflexion, on s'aperçoit qu'en réalité il faut les solliciter pour en tirer la preuve d'un ralliement personnel qui, à cette date, eût été prématuré. Assurément, les hostilités n'ont été conduites que par Octave, et n'ont tourné qu'à son profit et à sa gloire. Mais Antoine, de loin, en partageait la responsabilité, et aux termes des accords de Tarente, intervenus l'année précédente( (1), il avait passé à Octave, pour mener à bien cette entreprise commune, une partie de ses effectifs et plusieurs de ses officiers ${ }^{(5)}$.Messala était du nombre, voilà tout; et il n'a fait, en servant Octave, qu'obéir à Antoine. En vain, pour démontrer le contraire, alléguerait-on l'épisode, complaisamment raconté par Appien, où l'on voit Octave, coupé de son escorte et surpris par l'adversaire, n'échapper que de justesse à sa poursuite grấce à l'assistance et au refuge que, près d'Abala, dans le Bruttium ${ }^{(6)}$, il a trouvé sous la tente de Messala( $\left.{ }^{(}\right)$. Mais si Messala avait, ce jour-là, tué Octave fugitif et à peu près désarmé, il aurait assuré la victoire de Sextus Pompée et du mème coup trahi Antoine. En outre, à supposer,

(1) Fontaine, op. cit., p. 28.

(2) Hammer, op. cit., p. 27 , pense que le ralliement à Octave remonte jusqu'en to av. J.-C., mais il n'en invoque point d'autre indice que la séparation ophysiquen, à cette date, de Messala et d'Antoine; et pour lui, comme pour Fontaine, le signe tangible de cette rconversion, c'est fa guerre d'Octave contre Sertus Pompée qui le montre (p. 38-32). Syme, op. cit., p. $237_{-2} 38$, ne se prononce pas. (3) Nauloque est du 3 septembre; l'abdication de Lépide antérieure au 23 septembre 36 av. J.-C.; cf. Gelzer, P. W., X, c. 316-317.

(1) Sur ces accords, ef. Attilio Levi, Ottaviano Capoparte, Florence, 1933 , p. 70 et suiv.

(5) 120 navires (Appien, B. C., V, 95,396 ) avec, à leur tête, Statilius Taurus (ibid., 98, 408); cf. P. W., III, c. 2599 .

(6) Sur la position d'Abala, vers Bagnara, entre Nicotera et Scilla, ef. Huelsen, P. W., l, c. 12.

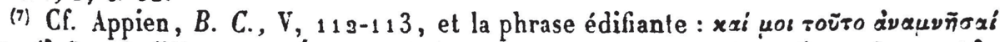

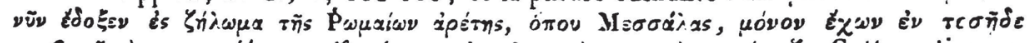

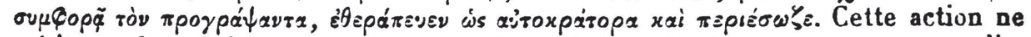
mérite ces hyperboles que parce que la réconciliation n’était pas encore accomplie. 
comme le prétend Dion Cassius, qu'Octave lui ait, dès lors, témoigné sa gratitude, en lui accordant, en surnombre, un siège dans le collège des augures (1), cette nomination, même si Dion ne l'a pas, comme je le crois, antidatée (2i, peut bien prouver qu'Octave cherchait déja à gagner le cœur de Messala; elle ne saurait établir que Messala s'était, pour autant, détaché d'Antoine. Aussi bien, tant que les deux triumvirs marchaient d'accord, ces sortes de défections n'avaient-elles point de sens; et Appien, sur l'anecdote de qui l'on s'appuie pour en admettre la réalité dès 36 avant J.-C., est précisément l'auteur qui, sans lui assigner un moment précis, l'a trop rapprochée du consulat de Messala, en 31 avant J.-C., pour ne point avoir pensé, lui-même, qu'elle n'avait dû s'accomplir que trois ou quatre ans p'us tard (3).

Pour écrire les chapitres 10 9-113 de son livre V, où la figure de Messala ressort en si belle lumière, Appien avait sûrement eu à sa disposition, soit les « histoires » de Messala (4), soit un auteur qui s'en était inspiré (3). De là, ces détails propres à grandir notre homme, l'importance donnée au sauvetage d'Octave en péril, et l'insistance avec laquelle Appien a fait un sort aux moindres allées et renues de Messala dans les semaines qui ont précédé l'écrasement, en Sicile, des forces hostiles à Octave ${ }^{(6)}$. Mais ne nous y trompons point : si l'auteur grec ne perd pas une occasion de mettre le personnage en relief, il ne songe nullement à faire état des récompenses que l'activité de Messala lui aurait obtenues d'Octave et il reste muet, notamment, sur l'augurat surnuméraire mentionné par Dion Cassius. Mais surtout, nulle part, il ne s'avise de le représenter comme un chef qu'Octave aurait chargé de mener ses hommes à la bataille.

Dans la première phase des opérations, le nom de Messala n'est pas eité. De son quartier général de Pouzzoles ${ }^{(7)}$, Octave avait prescrit, pour le $1^{\text {ox }}$ juillet 36 avant J.-C., une attaque générale et combinée de la citadelle pompéienne : tandis que lui-mème descendrait du nord en Sicile, Taurus, venu de Tarente, aborderait l'ile par l'est, et Lépide, appelé d'Afrique, par le sud-ouest : la Trinacrie serait démantelée simultanément sur ses trois côtés à la fois par les escadres et les légions des trois triumvirs ${ }^{(8)}$. Messala n'est point mentionné parmi les exécu-

(1) Cass. Dio, XIIX, $16,1$.

(2) Cf. infra, p. $107-108$.

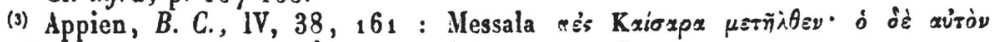

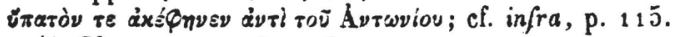

(6). Cf. supra, p. 96, n. 4.

(5) Cf. Soltau, Philologus, supp ${ }^{t}$ VII $(1899)$, p. 62 s.

(6) La source d'Appien étant favorable, en toute hypothèse, à Antoine (cf. Schwarz, $P$. $W$., II, c. 233 ), cet intérèt pour Messala serplique par son appartenance à la clientèle d'antoine.

(7) Et du Portus Iulius; of. Appien, B. C., V, 97 et et Hadas, Sextus Pompey, New-York, 1930 , p. 123.

(s) Appien, ibid. 
NOTES BIOGRAPHIQUES SUR Y. VALERIUS MESSALA CORVINUS.

tants de ce plan grandiose, peut-être parce que le plan devait avorter, en tout cas parce qu'il n'occupait encore qu'une situation subalterne.

Le 3 juillet 36 avant J.-C., la tempête avait soufflé sur les desseins d'Octave : tandis que Lépide n'avait pu qu'à grand'peine, et non sans pertes, opérer son débarquement au sud de Lilybée (1), Statilius Taurus avait été contraint de rentrer, avec des navires en moins, à sa base de Tarente; et Octave et Agrippa avaient été empéchés de déboucher du golfe de Velia (2). Sans Messala, ou avec Messala obscurément en sousordre, la grande offensive était manquée (3).

La sagesse eût peut-être consisté à la remettre à la belle saison de l'année suivante, et c'est apparemment ce qu'avait prévu Sextus Pompée et ce qui, partiellement au moins, explique son inertie (4). Mais Rome souffrait de la disette et la plèbe, malheureuse, affamée, n'eût point supporté, sans troubles graves, un aussi long ajournement. Octave décida de brusquer les choses et de recommencer le plus tồt possịble l'entreprise. Cependant que, sous la direction d'Agrippa, les arsenaux de Pouzzoles travaillaient à plein pour réparer en hâte les dégâts subis par les escadres ${ }^{(5)}$, il voulut communiquer à tous ses subordonnés sa propre ardeur, et il entendit se montrer partout à la fois : à Tarente, où il s'en alla, de prime abord, réconforter Taurus ${ }^{(6)}$, et sur toutes les positions stratégiques dont il prévoyait l'emploi et qu'il tenait à inspecter personnellement : Vibo (7), Co'umna Regia (8) et Scyllacium, où il se rencontra derechef avec Taurus (9). Or ce n'est qu'au cours de ces semaines de préparatifs, et du fait des circonstances, que, pour la première fois, nous voyons intervenir Messala, dans la plus importante; peut-être, des missions qui !ui échurent en cette guerre.

Pendant un de ces déplacements qui avaient éloigné Octave de Pouzzoles, et a'ors qu'Agrippa, son second, avait lui-même quitté la base pour monter dans les fùtaies d'alentour et presser le travail des bùcherons qui débitaient le bois nécessaire à la réfection et à la construction des navires, Messala eut à recevoir, sans doute comme le p'us élevé en grade des officiers présents ${ }^{(10)}$, la secrète visite qu'était venu faire à ses chefs le lieutenant de Sextus Pompée, Ménodore : en leur absence, c'est Messa!a

(1) Appien, B. C., V, 98 .

(2) Ibid.

(3) Cf. Hadas, op. cit., p. 125.

(4) Ibid., p. 126 .

(s) Appien, B. C., V, $9^{8}$.

(6) Appien, B. C., V, 99 .

(7) Appien, B. C., V, 103.

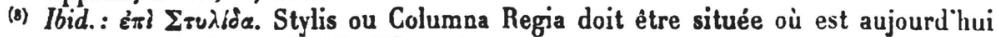
Cannitello, ef. Huelsen, $P$. W. IV, c. 603 .

(9) Appien, B. C., V, 103. Sur Scyllacium, Squillace, ef. Philipp, P. W., II, e. 921 et Hadas, op. cit., P. 129 ; n. 34 .

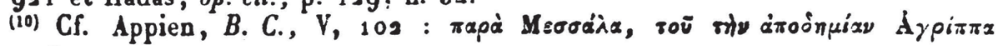
doเxอบัyтos. 
à qui Ménodore confia son intention de trahir et qui prit sur lui d'accepter en leur nom les propositions du transfuge (1). N'importe qui, sans doute, aurait agi de même à sa place; et le hasard l'avait bien servi en lui offrant cette brillante occasion de tenir la vedette; mais, tout de mème, ce n'était là qu'un hasard, et il ne parait pas que, dans la suite, Octave lui en ait su plus de gré que cela n'en méritait.

Lorsqu'en effet le branle-bas de combat fut à noureau donné, Messala, au lieu d'ètre appelé jamais en première ligne, dut toujours se contenter de commander, soit des cadres de conduite, soit des relais à l'arrière. Absent de la narration de Dion Cassius ${ }^{(2)}$, il n'est mentionné que deux fois dans le récit d'Appien, ou, pour parler plus exactement, qu'en deux situations du même service d'étapes, visées l'une au chapitre 103, l'autre dans les chapitres $105,109,110,11$ a et $113 \mathrm{du} \mathrm{V}^{\circ}$ livre :

$1^{\circ}$ Il reçoit d'Octave, alors à Vibo, mission d'amener de là deux des légions qui y étaient concentrées dans la région de Tauromenium (Taormina), où Lépide, déjà, s'était transporté, avec la prise de cette ville comme objectif ${ }^{(3)}$. Mais, après qu'il eut rempli ce mandat, Messala, au lieu de rester aux côtés de Lépide et à la tête des troupes qu'il lui avait conduites, doit, pour obéir aux ordres du général en chef, tourner le dos aux futurs champs de bataille siciliens, et revenir à Vibo auprès d'Octave (s).

$2^{\circ}$ Lorsqu'ensuite Octave se porte, de Vibo, à la rencontre de Statilius Taurus, qui stationnait à Scyllacium (Squillace), il emmène avec lui Messala et trois légions ${ }^{(5)}$. Il est possible que, pendant cette marche hors des vues et de l'atteinte de l'ennemi, Octave ait laissé, comme précédemment, le commandement de ces trois légions à Messala. Mais lorsqu'il eut effectué sa jonction avec la flotte de Statilius Taurus, et qu'ensemble, et avec toutes leurs forces, les deux chefs se furent avancés vers Leucopetra (cap dell' Armi) ${ }^{(6)}$, d'où Octave se proposait de descendre en Sicile, près de Tauromenium, avec autant de troupes que ses vaisseaux en pouvaient contenir ${ }^{(7)}$, Messala fut laissé sur la rive italienne avec le reste des soldats qu'on n'avait pu embarquer; et sa consigne

(1) Voir la suite du chapitre d'Appien.

(2) Cass. Dio, XLIX, 1-15. Il ne sera question de Nessala que la guerre terminée; cf. infra, p. 106 .

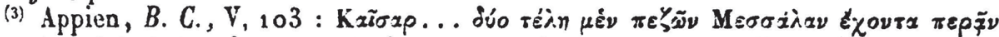

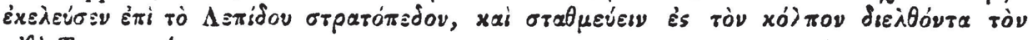

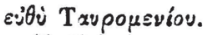

(s) Voir la note suivante.

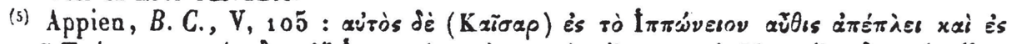

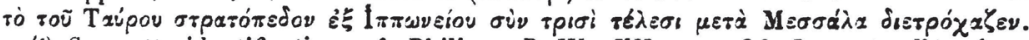

(6) Sur cette identification, cf. Philipp, $P$. W., XII, c. 2986 . Le texte d'Appien,

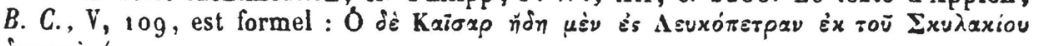

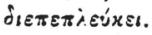

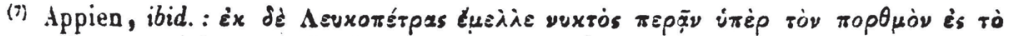

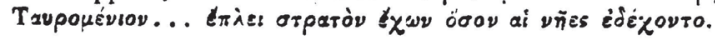


NOTES BIOGRAPHIQCES SLR M. VALERIUS YESSALA CORVINUS. 103

était d'y demeurer avec eux, inactifs, jusqu'au moment oủ les navires, redevenus disponibles, reviendraient les chercher (1). On sait, du reste, qu'il les y attendit vainement, et comment, au lieu de cingler vers les côtes de Sicile, c'est Octave, en train de les fuir, qu'il a recueilli dans le camp qu'il avait consciencieusement gardé (2). Malgré les éloges démesurés dont Appien a enguirlandé ce "fait divers», it ne semble pas qu'Octave ait aussi fort apprécié que sans doute, plus tard, Messala, en ses ehistoires", le service qui venait de lui être rendu; et tandis que lui-même, se ressaisissant arec une prompte énergie, méditait déjà sa revanche, il s'abstint d'y convier Messala, incontinent dépéché de Leucopetra à Pouzzoles pour y rassembler la $1^{\text {ro }}$ légion qui y avait été mise en réserve, et, de là, en convoyer les effectifs jusqu'à....Vibo ${ }^{(3)}$; et, comme Appien ne parlera plus de Messala dans la suite de sa rédaction( ${ }^{(1)}$, il est permis de se demander, non pas si ce jeune commandant d'étapes prit aux victoires finales de Nauloque et de Messine une part que ce silence a bien l'air d'exclure, mais s'il a seulement jamais remis le pied en Sicile.

En vérité, tout se passe comme si, d'un bout à l'autre de cette guerre, Octave, tout en traînant Messala après lui, avait voulu l'éloigner de toute fonction militante et véritablement active. Jusque sous l'Empire, Messala, et c'est à sa louange, ne cessera de professer une sorte d'idéal républicain qui lui a dicté sa démission de la préfecture de la Ville ${ }^{(5)}$ et à laquelle les Anciens ont rendu hommage ${ }^{(6)}$. Notamment, il n'a jamais caché le culte qu'il vouait à la mémoire de Brutus (i) : félicité, au soir d'Actium, par Octave, qui avait suivi avec autant de surprise que d'admiration les prouesses qu'il avait accomplies au cours de la journée, il revendiqua l'honneur de sa vaillance à Philippes ${ }^{\left({ }^{8}\right)}$, et $i$ l se refusa fièrement à désavouer le passé : il était resté, se borna-t-il à répondre, econséquent avec lui-même, puisqu'il avait pour principe de lutter toujours pour la p'us juste cause " ${ }^{(9)}$; et, sans pouvoir retenir ses larmes, il saisit l'occasion de présenter et recommander à Octave le Grec Straton, qui avait rendu à

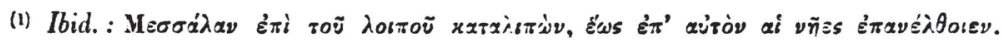
Slessala ne devait plus avoir grand monde, puisqu'au chap. 110 Appien énumire comme embarqués : 3 légions, כoo cavaliers, 1.000 vélites, 2.000 volontaires, sans compter les équipages.

(2) Cf. supra, p. 99, et Appien, B. C., V, $112-113$.

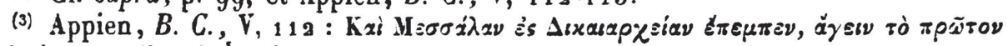

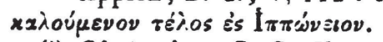

(s) Cf. Appien, B. C., V, 11 1-139.

(5) Cf. Tac., Ann., VI, 11,4 : ...quasi nescius exercendi; Saint Jérome, Chr., a. abr. 1991 : incivilem potestatem contestans.

(6) Cf. Tac., De or., XXI, 9; Ann., XI, 6, 4; Vell. Pat., II, 112 , 2; Quint., XII, $11,28$.

(7) Et de Cassius; ef. Tac., Ann., IV, 34, 6 : Messala Curvinus imperalırem sunn Cassium praedicabat.

(8) Cf. Plut., Br., XL, 9 et XLI, 2.

(1) Plut., Br., LIII, 1 . 
Brutus le suprème service d'aider ce noble vaincu à ne point survivre à sa défaite ( 1 . En 4 a avant Jésus-Christ, la cruauté d'Octave envers Brutus mort ${ }^{(i)}$ avait trop contrasté avec l'humanité dont Antoine avait fait preuve alors ${ }^{(3)}$, pour que, dans son ralliement au triumvivat, Messala n'ait point distingué entre les triumvirs : il s'est attaché à celui-ci, détourné de celui-là. De son côté, Octave, qui savait lire dans les cœurs (s), a dû témoigner une prudente réserve envers un homme qui se montrait aussi obstinément fidèlo à un souvenir entre tous détesté, et que les conjonctures de la politique n'ont longtemps rapproché de lui que par l'entremise de son partenaire au triumvirat. Ainsi, à la fin de 40 avant JésusChrist, au lendemain de la paix de Brindes(5), Messa!a avait soutenu de sa parole les prétentions d'Hérode à la royauté. qu'Octave et Antoine s'étaient entendus pour faire admettre au Sénat ${ }^{(6)}$; mais nul n'ignorait qu'en cette affaire l'initiative n'avait pas appartenu à Octave, et que Messala, ainsi d'ailleurs que son auxiliaire en ce débat, L. Sempronius Atratinus (7), n'avait été que le porte-parole d'Antoine ${ }^{(8)}$. De mềme, dans la guerre de 36 avant Jésus-Christ contre Sextus Pompée, cet ennemi du triumvivat tout entier, sa présence aux côtés d'Octave préjugeait si peu le fond de ses attachements, qu'à la fin des hostilités Messala n'obtint aucun des commandements qu'Octave s'empressa de conférer à un ancien partisan d'Antoine dont il considérait le dévouement comme désormais acquis à sa personne : Statilius Taurus.

Dans la période précédente, celui-ci s'était signalé par son zèle pour Antoine. C'est à Antoine qu'il avait dû d'être choisi pour légat du proconsul d'Afrique, quand C. Calvisius, par la grâce d'Antoine, eut été, à l'automne de 44 avant Jésus-Christ, investi de ce gouvernement ${ }^{(9)}$. C'est encore la cause d'Antoine, qu'au plus fort de la guerre de Modène, et nonobstant les condamnations fulminées contre l'ancien lieutenant de César, Statilius Taurus avait continué, avec C. Calvisius, de défendre sournoisement contre Cicéron, lequel, dans une lettre écrite aux environs

(1) Ibirt.; cf. Vell. Pat., II, 70; 4-5.

(2) Suét., Aug., XIIt, 3 : Nec successum victoriae moderatus est, sed capite Bruti Romam misso, ut statuae Caesaris subiceretur, in splendidissimum quemque captivum non sine verborum contumelia sizeviit.

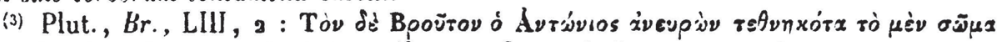

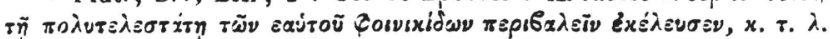

(4) Cf. Suét., Aug., LI, LIII, LVI, LXVI, LXVII, etc.

(s) Sur la date de cette pair voir mon livre: Virgile et le mystère de la IV' Eglogue, aì elle est fisée aur environs du 5-6 octobre 40 av. J.-C.

(6) Voir les récits concordants de Josèphe, $A$. J., XIV, 14 et $B$. J., I, 1'.

(7) Sur Atratinus, ef. Muenzer, $P$. W., $\mathrm{II}^{\wedge}$, c. 1367 .

(3) C'est ce que dit expressément Joséphe, A. J. XIV, 14, A, et $B . J ., I, I_{1}^{\prime}, \mathcal{A}$. 1)'ailleurs quand le Sénat eut ratifé la royauté d'Hérode, c’est par un grand banquet chez Antoine que fut fèté le nouveau roi (ibid.).

(9) Cic., Phil., III, 10, 36: voir le commentaire de Pallu de Lessert, Fastes, I. p. 54, et la note de Tyrrell et Purser, dans leur édition des Letires de Cicéron, VI, p. $7^{3}$. 
NOTBS BIOGRAPHIQUES SUR M. VALERIUS MESSALA CORVINUS. 105

du 20 mars 43 avant Jésus-Christ, vitupère la résistance agressive du Minotaure, c'est-à-dire de Calvisius et de Taurus réunis : offensione Minotauri, id est Calvisii et Tauri(1). Enfin, c'est toujours cornme le représentant d'Antoine que Statilius Taurus avait été désigné pour fair. contrepoids à Agrippa dans le consulat de 37 avant Jésus-Christ (2); et, enfin, ainsi que nous l'avons vu précédemment, c'est à lui qu'Antoine avait remis la flotte qu'en 36 avant Jésus-Christ il prêta à Octave contre Sextus Pompée(3). Mais Taurus était un homme nouveau( (1), sans lien avec les conjurés des ides de mars, sans convictions arrètées autres que !e progrès de sa carrière. A la faveur de la guerre de Sicile, il effectua la conversion qu'il jugeait profitable; et dès la fin des hostilités, il commença d'en être payé par une succession d'honneurs considérables.

D'abord, en quittant la Sicile, Octave le chargea d'achever la pacification de l'lle (5). Puis, satisfait de la manière dont Statilius Taurus s'était acquitté de cette táche, Octave lui accorda, au début de 35 avant JésusChrist, avec le glorieux titre d'imperator (8), le gouvernement des deux Afriques, où il devait rester près de dix-huit mois consécutifs (7), inaugurer la construction de l'enceinte de la nouvelle Carthage ${ }^{(8)}$ et, en une série de rencontres, battre les indigènes rebelles de telle sorte qu'à son retour à Rome il fut autorisé par Octave à célébrer le triomphe, le 3 o juin 34 avant Jésus-Christ ${ }^{(9)}$. En outre, se voyant astreint à interrompre sa victorieuse expédition en Illyrie pour assumer, dans Rome, son deuxième consulat, le $1^{\text {or }}$ janvier 33 avant Jésus-Christ, Octave plaça Statilius Taurus à la tête de son armée avec ordre d'en finir avec les Dalmates, et l'entreprise fut couronnée d'un plein succès que récompensèrent sans délai un énorme butin (10) et une seconde salutation impériale(11).

Evidemment, si tant de responsabilités et de pouvoirs accumulés manifestent le crédit dont Taurus jouissait auprès d'Octave, l'inaction à laquelle le triumvir d'Occident a simultanément réduit Messala prouve le peu de confiance qu'il lui accordait alors.

(1) Cic., Ad fam., XII, 25 (825 T. P.), 1 .

(2) Cf. C.I.L., $\mathrm{l}^{2}$, p. $65_{1}$.

(3) Cf. supra, p. 99 , n. 5 :

(1) Vell. Pat., II, 127, 1: Ut divus Augustus (usus est) M. Agrippa, et maxime ab en, Statilio Tauro, quibus novitas familiae non obstitit.

(5) Orose, VI, $18,32$.

(6) Cf. Dessau, I. S. L., 893 .

(7) Étant donnée la date de son triomphe; cf. infra, n. 9. Ce gouvernement est attesté, en dehors d'Orose, par Cass. Dio, XLIX, 14 .

(8) Tertullien, De palliv, 1 : Ubi moenia Statilius Taurus imposuit, sollemnia Sextius Salurninus enarravit. Je n'en tiens sur ce point à l'opinion courante; mais Gsell, Hist. anc. de $l^{\prime} .1 f r$., VIII, p. $17 \bar{j}$, rapporle cette inauguration à 4 ' 1 ar. J.-C.

(9) C. I. L., l', p. 50 .

(10) Cf. P. W., III", c. 2201.

(11) Cf. Dessau, I. S. L., 893 . Sur son commandement illyrien, ef. Appien, Ill., 28; Cass. Dio, XLIX, 38 et Orose, VI, 21. 


\section{III}

Cette inaction, les Modernes n'en ont pas voulu convenir. Ici, en interprétant à la lettre une phrase de Dion Cassius; et là, au contraire, en bousculant des textes qu'ils utilisent sans les avoir pesés et confrontés, ils affirment généralement qu'après avoir exterminé les forces de Sextus Pompée, Octave a marqué sa faveur à Messala en iui attribuant une doubie récompense : honorifique, avec l'augurat qu'il lui aurait alors attribué ; effective, avec le commandement dont il l'aurait pourvu, en 35 avant Jésus-Christ, dans sa première campagne d'Illyrie (1). Mais il n'est que d'examiner de près leurs documents pour s'apercevoir, d'une part, qu'un sacerdoce, si haut soit-il, n'implique aucune puissance réelle, et qu'au surplus la date à laquelle ils en placent la collation est discutable; d'autre part, qu'à les interpréter correctement, les témoignages à l'aide desquels ils se flattent de la démontrer s'opposent à la participation de Messala aux opérations militaires de 35-33 avant Jésus-Christ dans les pays illyriens.

Sur le premier point, du moins, l'assertion de Dion Cassius est formelle : après avoir énuméré, dans le chapitre 13 de son Livre XLIX, les distinctions dont le Sénat combla Octave après la guerre de Sicile, l'historien poursuit en ces termes au début du chapitre 14 : "Voilà ce qui fut décrété à César par le Sénat. Quant à lui, il inscrivit en surnombre parmi les augures le Valerius Messala qu'il avait condamné à mort dans les proscriptions et il concéda le droit de cité romaine aux

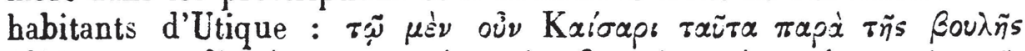

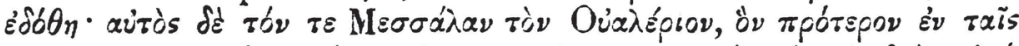

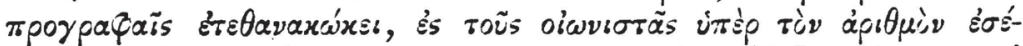

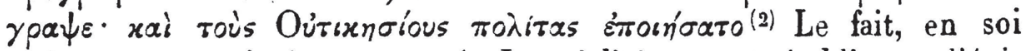
-même, ne saurait être contesté. La réalité en est établie par l'épitaphe, découverte à Rome, d'un affranchi de Messala, Philargyrus, qui, justement, lui servait d'appariteur en sa qualité d'augure : $M$ (arcus) Valeriu[s M(arci)] Messalae $\mid$ l(ibertus) Philarg(yrus) |viat(or) augur(2s) ${ }^{(3)}$; et, par ailleurs, elle semble s'accorder avec ce que nous entrevoyons de la carrière parallèle de Lucius Sempronius Atratinus, qui, appartenant, comme Messala, à la clientèle politique d'Antoine, avait été son émule, d'abord dans la défense de la couronne d'Hérode ${ }^{(4)}$. et ensuite dans la lutte contre Sextus Pompée ${ }^{(5)}$ : Atratinus, en effet, figure comme aug(ur)

(1) Cf. Fontaine, op. cit., p. 31-34, et Hammer, op. cit., p. 32-40.

(2) Cass. Dio, XLIX, 16,1 .

(3) Dessau, I. S. L., 4977 .

(i) Cf. supra, p. 104 .

(5) C'est le fait que Yluenzer a mis en lumiere, $P . W ., \mathrm{II}^{4}$, c. $136_{7}$, en renvoyant aux monnaies des villes siciliennes de Lilybée et d'Entella qui portent la légende 
NOTBS BIOGRAPHIQUES SUR x. VALBRIUS ressala CORTINUS. 107

sur des monnaies probablement émises en 36 ou 35 avant Jésus-Christ (1); et il est tentant de supposer qu'Octave avait pareillement traité les deux collaborateurs occasionnels qu'il tenait de la bonne volonté d'Antoine. Mais, à y regarder de près, le rapprochement écarte, plutôt qu'il ne l'appelle, la simultanéité de leurs dignités sacerdotales. Si la renommée de Messala a finalement éclipsé celle d'Arratinus, celui-ci, à l'époque du triumvirat, en laquelle nous nous plaçons, l'emportait, et de beaucoup, en influence et en dignité, sur celui-là. Atratinus avait neuf ans de plus que Messala (2); sa cooptation dans le collège des augures remontait à la paix de Brindes, ainsi qu'il résulte d'un fragment, rédigé en 40 avant Jésus-Christ, de leurs fastes corporatifs (3); et elle n'avait fait que préluder à une promotion bien plus importante : dès l'année suivante, en effet, et en vertu des stipulations de la paix de Pouzzoles conclue à l'été de $3_{9}$ avant Jésus-Christ ${ }^{(4)}$, sinon de celles de la paix de Brindes, en octobre 40 avant Jésus-Christ, Atratinus fut compris, par Octave et Antoine associés, dans la liste des futurs titulaires du consulat (5), qu'il gérera effectivement en 34 avant Jésus-Christ ${ }^{(6)}$. Selon toute probabilité, son élévation au sacerdoce, proclamée alors qu'il venait d'accomplir ses 33 ans, avait eu pour but d'annoncer et préparer son acheminement à la magistrature suprême, pour laquelle Messala ne sera désigné à son tour qu'en $3_{2}$ ou $3_{1}$ avant Jésus-Christ, à l'âge de 32 ans ${ }^{(7)}$. Si donc l'on veut maintenir un exact parallélisme entre l'avancement de Messala et celui d'Atratinus, c'est avec le même intervalle d'une année entre le sacerdoce et le consulat et, par conséquent de 33 plutôt que de 36 avant Jésus-Christ, qu'il faut dater la cooptation surnuméraire de Messala parmi les augures.

Aussi bien cette date, qui-coincide avec celle que nous finirons par assigner au ralliement de Messala à la personne d'Octave ${ }^{(8)}$, convientelle mieux également que celle de 36 avant Jésus-Christ au privilège qui, suivant Dion Cassius, aurait été, en même temps, accordé aux gens

Arparivov (Catal. of gr. Coins Sicily, g5 et 61,8 ), preuve que le personnage arait été préposé à la police de ces places insulaires comme Messala à celle des stations continentales de Vibo, et, par interim, de Pouzzoles. 515 .

(1) Cf. Muenzer, loc. cit., d'après Grueber, Coins of the Roman Rep., II, 501 et

(2) Puisqu'il est né en $7^{3}$ av. J.-C. ainsi qu'il résulte de Saint Jéròme, Chron., II, p. 143 -g Schöne, cité par Huenzer, op. cit., c. 1366.

(3) Cf. Dessau, I. L. S., $9348,3$.

(a) Sur cette pair, dite à tort de Misène, voir mon article de 1913 dans la $R$. A., et mon mémoire de la R. H., 1999, CLXI, p. 297.

(s) Cf. Nuenzer, loc. cit., c. 1367 , d'aprés les monnaies citées plus haut, dont les légendes associent à Antoine, impe(rator) ter co(n)s(ul) desi(gnatus)iter et ter, itratinus, non seulement augur, mais $\operatorname{co}(n) s(u l)$ desig(natus).

(6) Cf. C. I. L., I', p. 66.

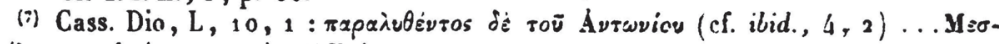

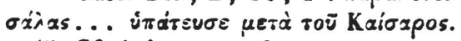

(8) Cf. infra, p. 116 . 
JÉ HỎMB GABCOPINO.

d'Utique (1). On ne discerne pas, en 36 avant Jésus-Christ, la raison pour laquelle Octave aurait tout d'un coup gratifié de la cité romaine ce municipe africain; la mesure s'explique, au contraire, de soi, si, postérieurement au rétablissement de l'ordre en Afrique proconsulaire, opéré en 35 et 34 avant Jésus-Christ par Statilius Taurus (2), Octave a voulu récompenser le concours que les habitants de la résidence gouvernementale (3) avaient apporté, contre le banditisme et la rébellion, au représentant de l'autorité romaine dans leur province (4).

Mais, dira-t-on, Dion Cassius est catégorique, et c'est à l'année 36 avant Jésus-Christ qu'il a tout ensemble rapporté, et l'extension de la civitas romana au municipium Uticense, et l'entrée de Messala dans le coilège augural. Il est vrai : seulement Dion Cassius n'en est pas à une confusion près. Il s'embrouille souvent dans ses fiches; et, pour ne citer qu'un exemple de son inexactitude, nous verrons tout à l'heure qu'il a antidaté de trois ou quatre années au moins l'action militaire de Messala contre les Salasses.

\section{IV}

Celle-ci, il est rrai, n'a pas été comprise par les modernes biographes de Messala, lesquels n'ont su la localiser, ni sur la carte, ni sur le calendrier. Pour tous, elle a eu pour théatre alpestre le pays d'Aoste alors tenu par des Gaulois turbulents que nos textes appellent effectivement Salasses et que les légions romaines avaient matés pour la première fois en 143 avant Jésus-Christ ${ }^{(5)}$. D'après les uns (6), elle s'est conjuguée avec l'expédition illyrienne d'Octave en 35-34 avant Jésus-Christ. D'après les autres ${ }^{(7)}$, elle l'a suivie, comme la conséquence de la part brilante que Messala avait prise, sous les ordres d'Octave, à la lutte contre les Iapydes. Or, à mon humble avis, les uns et les autres se sont fourvoyés.

Malgré qu'en aient ces érudits, les Salasses du Val d'Aoste n'ont rien à voir avec les Salasses que Messala a combattus. Assurément, la Realenzy-

(1) Cf. supra, p. 106 , n. 2.

(2) Cf. supra, p. 105 .

(s) Le premier proconsul dont nous soyons assurés qu'il ait résidé à Carthage est Pison en 70 ap. J.-C. (Tac., Ann., IV, 49-50); et, comme l'écrit Audollent, Carthage romaine, p. 50, il faut descendre jusqu'à Néron pour rencontrer un fait (Suét., Nero, XXXI; Tac., Ann., XVI, 1-3) qui soit de l'histoire de la ville mèmen.

(4) Exactement comme Claude (voir mon Marnc antique, p. 43) récompensera le municipe de Volubilis de son concours contre Aedemon; sur le municipium Iulium Eticense, cf. Gsell, Hist. anc. Afr. Nord, VIII, p. 197, n. 4.

(s) Liv., Per. LIII : Appius Glaudius Pulcher consul Salassos gentem Alpinain domuit.

(i) Fontaine, op. cit., p. 32 ; ef. Valeton, op. cit., p. 43.

(7) Hammer, op. cit., pp. 38-40. 
YOTES BIOGRAPHIQUES SUR M. FILERIUS MESSALA CORVIYUS.

clopiidie ne connait point d'autres Salasses que ceux du Val d'Aoste (1); mais dès qu'on examine les textes anciens où sont mentionnés des Salasses on se convainc de la coexistence, en des cantons montagneux fort éloignés l'un de l'autre, de deux peuplades différentes portant le mème nom (2). Après tout, des doub!ets de ce genre ne sont pas rares sur l'aire des migrations celtiques; et puisqu'il y a eu des Andécaves à Mantoue et à Angers, des Boïens sur les rives de la Loire et sur celles du Pó, et des Sénons aux bords de l'Aube et de l'Adriatique, il n'y a pas lieu d'ètre surpris de trouver deux groupes de Salasses en deux régions des Aipes.

- Pour la confusion de nos érudits, le malheur a voulu que Messa!a ait eu affaire aux deux. D'une part, en effet, Strabon nous raconte que les Salasses du Val d'Aoste, maîtres jaloux des cols dont ils commandaient l'accès ${ }^{(3)}$, par ailleurs soucie ux d'écarter l'étranger de la richesse de leur so: (4), et de surcroit pillards invétérés, eurent l'occasion, après avoir rançonné Decimus Brutus, d'exploiter indignement Messala (5). D'autre part, dans ses Illyrica, Appien nous a peint, des Salasses que Messala réduisit par la famine ${ }^{(6)}$, un portrait dont plus d'une couleur rappelle celles de Strabon : ils habitaient, dans les Alpes, des hauteurs difficilement accessibles ${ }^{(i)}$ et ils mettaient en coupe réglée qui prétendait montr $\mathbf{r}$ aux défilés dont ils étaient les maitres ${ }^{\left({ }^{(}\right)}$. De là à conclure qüici et là ce sont les mêmes Salasses, il n'y a qu'un pas; et il a été franchi, non seulement par Fontaine et Hammer, mais par des historiens comme Guglielmo Ferrero ${ }^{(9)}$ et Camille Jullian ${ }^{(10)}$. Mais leur erreur est aussi certaine qu'elle est répandue ${ }^{(11)}$; et dès que l'on compare les textes qui nomment les Salasses, la dualité de leurs peuplades saute aux yeux.

Les Salasses de Strabon exploitent des gisements aurifères ${ }^{\left({ }^{12}\right)}$; ceux d'Appien, des mines de sel ou des marais salants ${ }^{(13)}$. Les Salasses de Strabon sont inclus dans sa description de la Gaule. Ceux d'Appien sont incorporés à l'Illyricum, avec les Iapydes, les Dalmates, les Segestani de Siscia (Siseg) et les Paeones, qui, de tous les Illyriens, sont les

(1) Cf. Philipp, P. W., I^, c. $18 \div 9$.

(2) Signe, peut-être, d'une parenté ethnique rattachant à un tronc commun ces rameaux divergents.

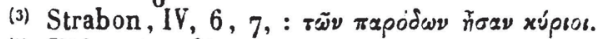

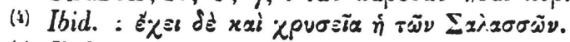

(s) Ibid.

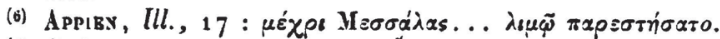

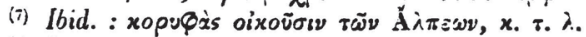

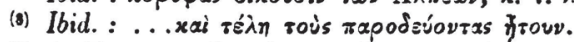

(9) G. Ferrero, Grandeur et Décadence de Rome, IV, p. 152.

(10) C. Jullian, Histoire de la Gaulp, IV, p. 58, n. 5 et כa.

(ii) Est seul à ne l'avoir point partagée un savant américain, Frucs Frarero, dans un livre The valley of Aosta, New-York, 1910, p. 125, que je ne connais que par la citation d'Hammer, op. cit., p. 37.

(12) Cf. supra, n. 4.

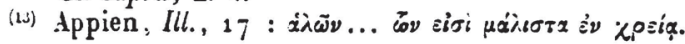


JÉRôME CARCOPIYO.

plus éloignés d'eux ${ }^{(1)}$. Comme les Paeones occupent le Rhodope et s'étendent jusqu'au Strymon(2), c'est à l'autre extrémité de la chaîne illyrienne qu'il faut les chercher, quelque part en direction de la Carniole, de l'Istrie ou du Frioul (3); mais où que nous songions à loger leurs nids d'aigles, il nous est évidemment interdit de les transporter à l'ouest de l'Italie, jusqu'à la frontière des Gaules. Enfin et surtout, Strabon et Appien nous en ont assez dit sur la succession des faits' relatifs à ces Salasses pour que nous soyons forcés de les organiser en deux séries radicalement distinctes. Chez Appien, Octave a d'abord chargé Antistius Vetus de mettre à la raison les Salasses d'Illyrie; puis, Vetus n'y ayant qu'imparfaitement réussi, Octave s'est reposé de ce soin sur Messala (4). Chez Strabon, Messala n'a point eu à guerroyer contre les Salasses de Gaule : ayant été assez mal inspiré pour hiverner dans leur voisinage, il a seulement dû leur payer le prix fort pour le bois de chauffage qu'ils lui ont fourni pour ses troupes ${ }^{(5)}$; et Auguste ne s'est ému de leurs rapines qu'après en avoir été personnellement victime ${ }^{(6)}$ : alors il a commis Terentius Varro à leur extermination (7). Ce général l'a poursuivie avec une cruelle vigueur : il a vendu comme esclaves 36.0 o o Salasses et, sur l'emplacement de son camp, il a fondé la colonie "Augusta" (3), dont le souvenir survit dans le nom moderne d'Aoste.

Au surplus, en séparant les destins des deux peuplades homonymes, on a belle à fixer l'année où s'est produit chacun des événements qui les ont remplis.

Pour les Salasses de Gaule, le calcul est vite fait. Dion Cassius place en a 6 avant Jésus-Christ les pilleries auxquelles ils se sont livrés sur les bagages d'Auguste, qui, marchant à travers les Gaules à son expédition contre les Cantabres, passa, par le col du Saint-Bernard, de la vallée de la Doire Baltée dans celle de l'Isère; et en 25 av. J.-C. la répression consécutive de Terentius Varro ${ }^{(9)}$. De son côté, Strabon nous apprend que la rançon, prélevée par les Salasses gaulois sur Decimus Brutus, l'avait été lorsque celui-ci se dirigeait de Modène sur la Transalpine ${ }^{(10)}$ :

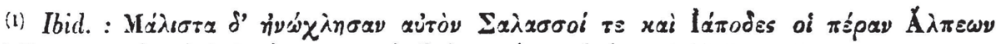

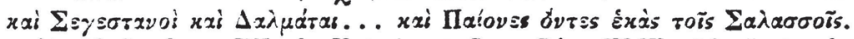

(3) Cf. Strabon, VII, fr. Vat. 4 , et Cass. Dio, XLIX, 36, 5 et suiv.

(3) C'est dans la mème direction que nous oriente la phrase de Cass. Dio, XLIX,

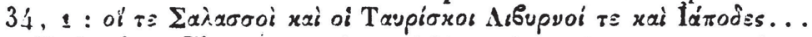

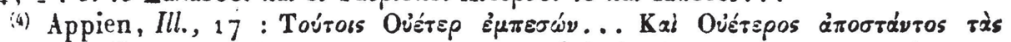

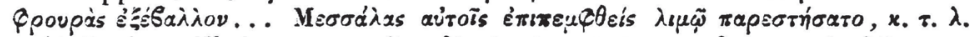

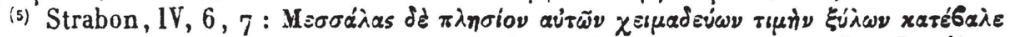

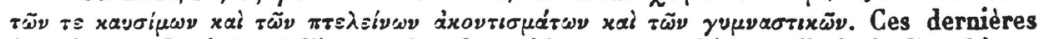
fournitures, destinées à l'instruction des soldats, prouve bien qu'il s'agit d'un hivernage militaire.

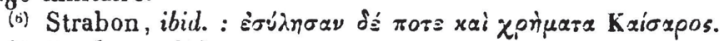

(:) Strabon, ibid.

(8) Strabon, ibid.

(9) Cass. Dio, LIII, 25.

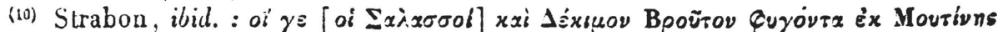


NOTES BIOGRAPHIQUES SUR Y. VILERIUS MESSALA CORVIYUS.

elle remonte, par conséquent, après la fin de la guerre de Modène, à 43 av. J.-C. Strabon ne nous a pas indiqué l'époque des désagréments que les Salasses gaulois causèrent à Messala. Mais il les intercale entre la mésaventure de Decimus Brutus et celle survenue à Auguste, entre 4 s et 26 av. J.-C., par conséquent; et il lies rattache à un hivernage de Messala qui se rapporte nécessairement à la campagne de Gaule sanctionnée par son triomphe du 25 septembre 27 av. J.-C. (1). D'où i! suit que Messala eut maile à partir avec les Salasses gaulois au cours de l'hiver 28-27 av. J.-C.

En ce qui concerne les Salasses iliyriens, la question de savoir quand Messala les a réduits par la famine est un peu plus compliquée; mais la vérité est relativement aisée à dégager de la contradiction des témoignages.

Suivant Dion Cassius, ce résuitat a été acquis après les victoires remportées en 34 av. J. C. par Fufius Geminus ${ }^{(2)}$ et avant ceiles remportées en 33 av. J.-C. par Octave et Agrippa sur les Dalmates ${ }^{(3)}$; et c'est, naturellement, cette chronologie qui a induit Hammer à considérer la mission de Messala comme. la suite de sa participation méritoire aux opérations antérieures ( ${ }^{(4)}$. Mais ce vague arrangement des faits est démenti par la précision des renseignements que nous devons à Appien. İ nous en tenir au schéma circonstancié des Illyrica, la soumission des Salasses illyriens s'est effectuée en trois temps : une première période où Antistius Vetus, les ayant cernés et forcés, a cru en avoir terminé avec eux ${ }^{(5)}$; une seconde période où les Salasses illyriens, s'étant empressés, dès que Vetus leur eut tourné le dos, de chasser les garnisons qu'il leur avait imposées, ont récupéré une indépendance de fait ${ }^{(5)}$; une troisième, et dernière, oủ Octave a vengé leurs affronts par le châtiment que Messala leur a infligé en son nom ${ }^{(7)}$. Appien n'a point spécifié les dates auxquelles se sont succédé ces phases de leur soumission; mais il nous a marqué un repère qui nous permet de traduire en valeur absolue sa chronologie relative. Il attribue aux menaces du conflit qui allait éclater entre lui et Antoine la rémission dont fit preuve Octave envers les rebelles entre le départ d'Antistius Vetus et l'arrivée de Messala sur leur

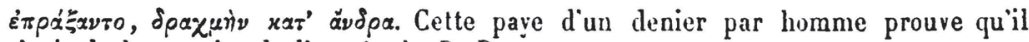
s'agit de la retraite de l'armée de D. Brutus.

(1) Cf. supra, p. 97, n. 4 .

(2) Cass. Dio, YLIX, $38,1-3$.

(3) Ibid., 4. C'est pourquoi Klebs, P. IV., I., c. 2558 , a daté de 33 av. J.-C. l'action de Vetus.

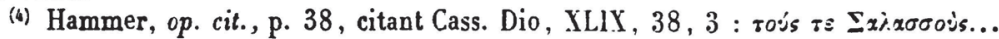

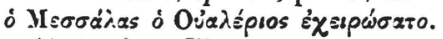

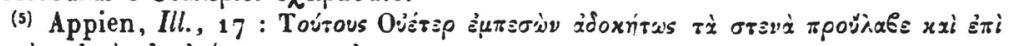

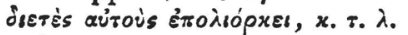

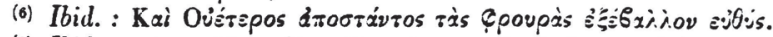

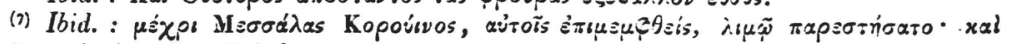

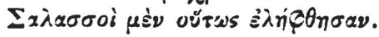


JÉRÔYE CARCOPIYO.

territoire (i). Il n'en faut pas davantage pour nous assurer : $1^{\circ}$ que les succés de Vetus ont été obtenus en 33 av. J.-C.; $2^{\circ}$ que l'insolence des Salasses illyriens s'est donné libre cours dans la période où Octave était occupé à préparer, puis à mener la lutte contre Antoine et Cléopàtre, entre 32 et 30 av. J.-C., par conséquent; $3^{\circ}$ que l'envoi de Messala contre ces insurgés n'a pas eu lieu avant la fin de cette grande guerre, c'est-à-dire qu'il est postérieur à la fois à la victoire d'Actium, en $3_{1}$ av. J.-C.. et à la prise d'Alexandrie, en 30 av. J.-C. Sur ce dernier point, les allusions plus ou moins floues de Dion Cassius sont démenties par la relation sommaire, mais circonstanciée, d'Appien; et celle-ci rompt définitivement le lien que les biographes de Messala ont aujourd'hui accoutumé de tendre entre les expéditions d'Octave, en 35-33 av. J.-C., dans les pays illyriens, et celle de Messala chez les Salasses d'Illyrie, en $2 g$ av. J.-G. au plus tót.

Essayera-t-on de le renouer, comme y a tấché Hammer ${ }^{(2)}$, en faisant état des vers du Panegyricus Messalae où l'auteur inconnu de ce plat poème, s'adressant à son héros, s'écrie avec emphase : «Non, ma muse ne doit pas errer au hasard de tes exploits ; je veux chanter ceux dont j'ai été témoin; j'atteste la défaite des vaillants lapydes; j'atteste la déroute des Pannoniens perfides que tu as rejetés en désordre sur les glaciers des Alpes; j'atteste les Arupini surpris sur leurs champs misérab!es...

....testis mihi victae
Fortis Iapydiae miles, tesis quoque fallax
Pannonius, gelidas, passim disiectus in Alpes,
Testis Arupinis et pauper natus in arvis ${ }^{(3)}$

Mais c'est là une tentative désespérée. Elle se heurte au silence d'Appien, qui, après avoir enflé à l'excès le rôle de Messala dans la guerre de Sicile ${ }^{(4)}$, n'en a point cité le nom parmi les généraux qui accompagnèrent Octave en Illyrie, tels qu'Agrippa, Claudius Nero, L. Scaevola(5), M. Heivius ${ }^{(6)}$ et Statilius Taurus, auquel, comme en Sicile, il confia son armée lorsqu'il regagna l'Italie (7). Mais surtout elle est en opposition avec les lois du genre de la pièce qu'elle invoque. Si, en Illyrie, Messala avait été subordonné à Octave, son panégyriste n'aurait pu, sans incon-

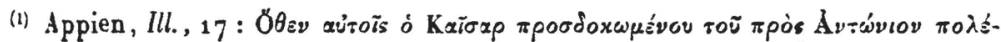

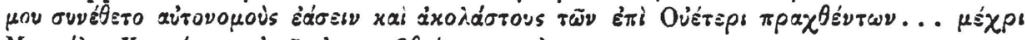

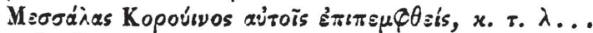

(2) Hammer, op. cit., p. 35.

(3) [Tib.], IV, 1, 107-1 10. Les Arupini situés près de Vital (cf. P.W., II, c. 1491) nous rapprochent de l'emplacement présumé des Salasses.

(s) Cif. supra, p. 99-100.

(s) Cf. Appien, Ill., 20: les copistes ont estropié les noms propres que Schweighauser a restitués.

(6) Appien, ibid., 21.

(7) Ihid., 37 ; cf. Cass. Dio, XLIX, 38, 4. 
NDTES BIOGRIPHIQUES SUR Y. VALERIUS MESSILA CORVIYUS.

venance, lui tresser ainsi des couronnes dérobées à son chef; et si, comme il est normal, il a entendu célébrer des hauts faits qui avaient été accomplis par Messala, alors que celui-ci commandait seul, et dont la gloire lui appartenait en propre, force nous est de reconnaître, sous ses hyperboles, la campagne, modeste et isolée, que Messala, en 29 av. J.-C., a dirigée, à travers l'Illyrie, contre les Salasses d'Appien. Il est paradoxal que le poète ne les ait pas nommés dans son énumération rhétoricienne; mais leur peuplade obscure, dont le nom, à cause des Salasses gaulois, prètait à équivoque, lui a sans doute paru peu digne de sa Muse; et s'autorisant de ce qu'Octave avait certainement délégué à Messala ses pouvoirs de surveillance et de police sur toutes les terres d'Illyrie récemment soumises qu'il traverserait pour parvenir jusqu'à eux, il a préféré jeter à tous les échos les sonorités connues d'ethniques plus retentissants, et paraphraser à sa manière l'information que nous' lisons chez Dion Cassius et suivant laquelle Messala avait réduit les Salasses et les autres tribus illyriennes qui s'étaient révoltées avec eux :

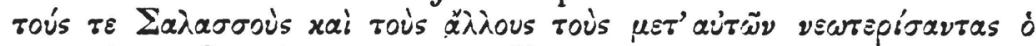

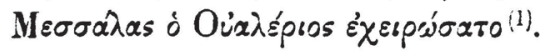

Maís alors, puisque Messala n'a pas accompagné Octave en Illyrie, comment donc a-t-il employé son temps pendant les années qui ont immédiatement suivi la guerre de Sicile? Tout porte à croire que, délaissé par Octave à la fin de 36 av. J.-C., il a bientôt profité de l'éloignement de celui-ci pendant les expéditions illyriennes pour rejoindre en Orient le triumvir auquel il avait engagé son dévouement personnel et qu'au moins jusqu'à l'automne de 34 av. J.-C. il a séjourné auprès d'Antoine, à Alexandrie.

$1^{\circ}$ De la probabilité de ce voyage, il existe un indice dans un document contemporain : la dixième pièce du livre I ${ }^{\text {or }}$ des Satires d'Horace. Le livre a paru en 34 av. J.-C.; et, de l'avis de Lejay, la dixième satire a été composée peu avant la publication de l'ensemble, c'est-à-dire en 35 av. J.-C. Messala y est deux fois l'objet de citations flatteuses d'ordre littéraire, et néanmoins fort instructives pour l'histoire. Aux vers 28-29, se moquant des orateurs qui mélangent avec affectation le grec au latin dans leurs harangues, Horace leur oppose l'exemple contraire de Pedius et de Corvinus :

$$
\text { Cum Pedius causas exsudet Poplicola atque Corvinus....; }
$$

et deux conséquences, jusqu'à présent inaperçues, découlent de cette

(U) Cass. Dio, XLIX, 38, 3. Ceci lève les difficultés signalées par Sıme, op. cit., p. 339 . 
JÉRÒME C.IRCOPIYO.

mention apparemment banale. La première, c'est qu'au lendemain de l'écrascment de Sixtus Pompée, Horace ne songe pas du tout aux actions d'éclat par lesquelles Messala se serait signalé dans la guerre de Sicile : son renom d'orateur est le seul dont le poète se soucie, comme si sa g!oire ne tenait toujours qu'à son éloquence; et il y a là une confirmation indirecte de l'effacement militaire où Octave avait relégué Messala. La seconde conséquence, c'est que le présent du subjonetif exsudet implique la présence de Messala au barreau de Rome dans le temps qu'écrivait Horace; et $\mathrm{du}$ même coup est vérifiée mon affirmation que Messala fut écarté par Octave des états-majors qui l'escortaient à cette date en Illyrie. Il est vrai qu'en même temps est exclue l'idée que Messala se fût déjà mis en route pour rejoindre Antoine. Mais l'autre passage de la même satire nous laisse prévoir cette éventualité et nous enveloppe, si je puis dire, de l'atmosphère d'un prochain ralliement.

A la fin du morceau, Horace énumère les bons juges par lesquels il lui plairait d'ètre lu et apprécié : «Je puis », dit-il, «déposant toute ambition, te nommer, Pollion, et toi aussi, Messala, ainsi que ton frère, et rous, en même tẹmps, Bibulus et Servius, et avec vous, toi, sincère Furnius, et plusieurs autres, doctes comme vous et comme vous mes amis, que, par sage propos, je passe sous silence :

Ambitione relpogata te dicere possum

Polio, te, Messalla, luo cum fratre simulque

Vos Bibule et Servi, sinul his te, candide Furni,

Complures alios, doctos ego quos et amicos prudens praetereo (')"

La sagesse finale dont se flatte Horace, en ses omissions volontaires, ne se comprend bien que si Horace n'a point voulu appeler l'attention sur des amis qui étaient a!ors trop mal vus du pouvoir pour ne point le compromettre, et eux avec lui, par des citations inopportunes, et l'on conçoit que son énumération ne trahisse, comme il s'en vante - ambitione relegata -, «aucune arrièro-pensée ambitieuse ${ }^{(2)}$, aucun esprit d'intrigue $^{(3)}$, si les critiques dont il recherche le suffrage n'étaient point, officiellement, trop bien en cour. La liste qu'il en a dressée dans la Rome gouvernée par Octave ne comporte, en effet, et à l'exception du Servius dont la personnalité est trop indistincte pour qu'on ose en tirer argument, que des partisans d'Antoine : Asinius Pollion, qui plus tard, lorsque la guerre aura éclaté entre les deux rivaux, aura le courage de résister aux sommations d'Octave et de maintenir à ses risque et péril une neutralité pleine de déférente gratitude pour Antoine (s); - Lucius Calpurnius Bibulus, un ancien vaincu de Philippes passé au service

(1) Hor., Sat., I, 10,8 s- 88.

(-) Traduction de Lejay, en note de son édition.

(3) Traduction de Villeneuve, en note de son édition.

(6) Vell. Pat., II, 86, 3. 
d'Antoine et nommé par lui, en 34 av. J.-C., au goisvernement de Syrie, qu il conservera jusqu'à sa mort, survenup en 3 z av. J.-C.(1); Caius Furnius, dont l'attachement à Antoine s'était affrmé dans la guerre de Pérouse, qu'Antoine avait envoyé en Afrique en 39 av. J.-G. et qui, parti aver lui en Orient. reçut de lui, en 35 av. J.C., la province d'Asis. enfin le demi-trère de Messala, comme lui un revenant de Pbilippes, ce Lucius Gellius Publicola qui devait rester fidèle à Antoine jusquà Actium, où il a commandé une aile de sa flotte (3).

En vérité, Messala se trouve, dans la prosopopée d'Horace, en p'ein milieu d'r. Antoniens:; entouré de partisans qui, abstraction faite de Pollion, demeuré à Rome, semblaient tous, par leur exemple, l'inviter à rallier leur chef à son tour.

$2^{\circ}$ La preure que Messala ne tardera pas à les imiter réside dans la notice sur Messala qu'Appien a insérée au livre IV de ses Guerres civiles. D'une part, y est-il écrit, Messala demeura étroitement lié à Antoine jusqu'au moment où, Cléopâtre ayant pris sur le triumvir un trop grand ascendant, il le tança pour cette lâche conduite et embrassa le parti

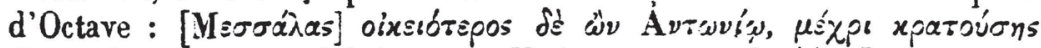

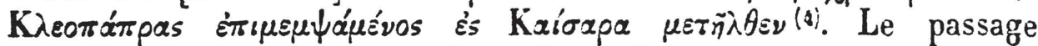
évoque une scène entre les deux hommes et cette scène suppose leur rencontre. D'autre part, Appien ne marque pour ainsi dire aucun temps d'arrêt entre ce retournement de Messala et la faveur qui lui valut d'être désigné comme consul à la place d'Antoine ${ }^{(5)}$. Le scandale qui a exaspéré Messala, c'est, à n'en pas. douter, à l'automne de $\mathbf{3 4}$ avant Jésus-Christ, le triomphe d'Alexandrie, où Cléopâtre parut en reine des légions romaines, et la seconde des donations qui démembrèrent l'empire au profit de ses enfants ${ }^{(6)}$. Entre le retour en Italie de Messala, écœuré par ces abandons, et sa désignation comme consul, se place, en 33 avant Jésus-Christ, sa nomination en surnombre dans le collège des Augures. Dion Cassius, ainsi que je crois l'avoir démontré, a antidaté la cooptation (7), mais, malgré lui, il l'a rapprochée de la désignation consulaire, en enveloppant les deux faits d'un commentaire identique et en exprimant, de l'un comme de l'autre, un étonnement qui se serait apaisé de l'un à l'autre si plusieurs années les avaient séparés (3).

(1) Cf. les références dans Muenzer, P. W., III, c. $1367-1368$.

(2) Cf. les références dans Groag, P. W., VII, c. 3;6-377.

(3) Cf. les références dans Juenzer, $P$. W., Vill, c. $1004-1005$.

(3) Appien, B. C., IV, 38, 161 .

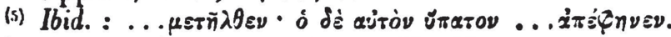

(6) Cf. Stähelin, $P . W ., X I$, c. $763-765$.

(7) Cf. supra, p. 106-1 08 .

(8) Comparer les deux passages :

Cass. Dio, XLIX, 16,1 :

Cass. Dio, L, 10, 1 :

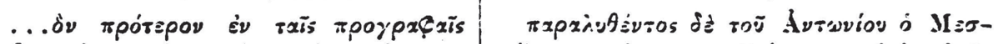

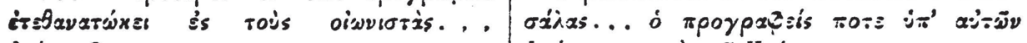
غंóxpops.

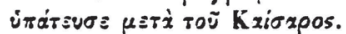


$3^{\circ}$ Nous arons una confirmation de ce processus de l'évolution de Ilessala dens lo souverit qui est parvenu jusquà nous des pamphlets qu'il a rédigés contre Antoine. Lo texte er est perdu; mais les titres et le sens en subsistent pour notre édification. L'un s'appelait le De Antonii statuis (1), l'aulre to Contra Antonii litteras ${ }^{(2)}$. Du premipr provient sûrement lo passago visé par Pline où Antoine est moqué de se servir, à la cour de Cléopitre, de pots de chambre en or ${ }^{(3)}$. Dans le second était probablement contée l'anecdote, promise à une si grande célébrité, de Cléopátre faisant fondre une perle d'une valeur de dix millions de sesteres dans un breuvage qu'elle avala d'un trait (\$). En tout cas, comme Pline a compris Nessala parmi les autrurs quil a consultés pour composer le livre IX où il la rapporte, i! n'est pas douteux que ce ne soit Messa!a qui l'ait rapportée d'Alixandrie. Ces historiettes relevaient de la propagande organisée par Octave en réponse aux lettres virulentes qu'Antoine répandait contre lui en Ita'is; et cette propagande a été d'autant plus efficace qu'elle a utilisé les dires d'un témoin oculaire des turpitudes qu'elle dénonçait à l'indignation des foules. Après avoir donné ce gage précieux de dévouement à Octave, Messala ne pouvait que lui inspirer confiance; et l'on s'explique pourquoi, en signe de sa bienveillante satisfaction, Octave ait, dès 33 avant Jésus-Christ, inscrit Messala dans un collège augural qui, pourtant, était au complet ${ }^{(5)}$; que, l'année suivante, il l'ait désigné pour remplacer, au consulat, Antoine ${ }^{(6)}$, que Nessala avait abandonné sans retour; et qu'enfin, sûr désormais de lui, il lui ait, pour la première fois, en 31 avant Jésus-Christ, confié une fonction militaire active et subordonné, sous le commandement supérieur d'Agrippa, une des escadres de la flotte qui, le a septembre, vainquit à Actium(i).

Après quoi, Messala suivit Octave en Égyte et reçut de lui les trois commandements successifs qui, croissant d'importance d'année en année, devaient, d'Asie dans les Balkans, puis d'Illyrie en Gaule, le conduire de proche en proche jusqu'au triomphe ${ }^{(8)}$ : en 30 avant Jésus-Christ, contre les gladiateurs rebelles d'Antioche (9); en 29 avant Jésus-Christ, contre les Salasses illyriens ${ }^{(10)}$; en 28 et 27 avant Jésus-Christ, contre les Aquitains (11). Mais avec cette dernière entreprise, nous reprenons pied sur un terrain dès longtemps connu; et je me bornerai, en terminant, à

(1) Cité par Charisius, I, $104,18$.

(9) Cité par Charisius, I, 139,7 .

(3) Pline, V. II., XXYIII, 5o : Messala orator prodidit Antonium triumurum aureis usum vasis in omnibus obscenis desideriis...

(4) Pline, N.H., IX, 119 .

(s) C.f. supra, p. 106.

(6) Cf. supra, p. 97, n. 3 .

(7) Appien, B. C., IV, 38, $15_{1}$

(8) Ci. supra, p. 97, n. 4 .

(9) Ciss. D10, LI, 7, 6 et 7

(10) Cf. supra, p. 112-113.

(u) Cf. supra, p. 111. 
NOTES BIOGRAPHIQUES SUR Y. VILERIUS MESSILI CORVIYUS. 117

résumer dans un tableau d'ensemble les conciusions de détail auxquelles cette modeste enquête a abouti :

4. av. J.-C.. : Messala, vaineu à Philippes, et pardonné par les triumvirs. se range dans le parti dintoine.

40 av. J.-C. : Messala, au nnm d'Intoine, patronne la cause d'Hérode.

36 av. J.-l. : Vessala est prèté par Antoine à Octave qui ne l'emploie que dans les services détapes de li guerre de Sicile contre Sextus Pompée.

35 av. J.-C. : Messala, après avoir repris sa place au barreau romain, cherche à rejoindre Intoine en Orient.

34 av. J.-C. : Messala séjourne à Alexandrie arec Antoine, puis s'y brouille avec lui.

33 av. J.-C. : Messala, rentré en Italie, passe au service d'Octave et de sa propagande et est fait augure en surnombre.

32 av. J.-C. : Messala est désigné pour le consulat de l'année suivante.

$\mathbf{3}$ av. J.-C. : Messala, consul, participe à la victoire d'Actium.

30 av. J.-C. : Messala est envoyé par Octave contre les gladiateurs d'Antioche. 29 av. J.-C. : Messala est envoyé par Octave contre les Salasses illyriens.

28-2j ar. J.-C. : Messala, qui hiverna à proximité du Val d'Aoste, rétablit l'ordre en Gaule, ce qui lui mérita le triomphe, le $25 \mathrm{sep}-$ tembre 27 av. J.-C.

Jérôme Carcopino. 